\title{
PREPROJECTIVE ALGEBRAS AND MV POLYTOPES
}

\author{
PIERRE BAUMANN AND JOEL KAMNITZER
}

\begin{abstract}
The purpose of this paper is to apply the theory of MV polytopes to the study of components of Lusztig's nilpotent varieties. Along the way, we introduce reflection functors for modules over the non-deformed preprojective algebra of a quiver.
\end{abstract}

\section{INTRODUCTION}

Let $\mathfrak{g}$ be a simply-laced semisimple finite dimensional complex Lie algebra. Part of the representation theory of $\mathfrak{g}$ is described by Kashiwara's combinatorics of crystals [15. On the algebraic side, crystals are implemented by special bases in representations, for instance, Lusztig's canonical and semicanonical bases [18, 20, 23. On the combinatorial side, a crystal can be realized by several models, each of which provides a concrete access to it; as an example, Anderson [1] and the second author's [12, 13. MV (Mirković, Vilonen) polytopes form a model for the crystal $B(-\infty)$ of the positive part $U(\mathfrak{n})$ of $U(\mathfrak{g})$.

The purpose of this paper is to apply the theory of MV polytopes to the study of the semicanonical basis. This basis arises through a geometric realisation of $U(\mathfrak{n})$ by means of constructible functions on certain varieties $\Lambda(\nu)$, called Lusztig's nilpotent varieties. Concretely, these varieties parametrize representations of the preprojective algebra constructed using the Dynkin graph of $\mathfrak{g}$. We are thus led to investigate the relations between these varieties $\Lambda(\nu)$ and MV polytopes.

Our method is the following. For each chamber weight $\gamma$, we construct a module $N(\gamma)$ over the preprojective algebra. Then we define a constructible function $D_{\gamma}$ on the nilpotent varieties whose value at a point $T \in \Lambda(\nu)$ is $\operatorname{dim} \operatorname{Hom}(N(\gamma), T)$. On an irreducible component $Z$ of a nilpotent variety, the functions $D_{\gamma}$ admit generic values. We prove that these generic values form the BZ (Berenstein, Zelevinsky) datum of an $\mathrm{MV}$ polytope, denoted $\operatorname{Pol}(Z)$. The resulting bijection $Z \mapsto \operatorname{Pol}(Z)$ defines a labelling of the semicanonical basis by MV polytopes, and hence by the crystal $B(-\infty)$.

This indexation of the semicanonical basis by $B(-\infty)$ is not new; indeed it was proved by Kashiwara and Saito [16] (and we use their result). However, an important property here is the fact that the MV polytope of an element $b \in B(-\infty)$ geometrically packs together all the Lusztig parametrizations of $b$. Therefore our result can be seen as the combinatorial facet of the relation between the semicanonical basis and the PBW (Poincaré, Birkhoff, Witt) bases of $U(\mathfrak{n})$. A remarkable fact is that this relation holds even when the reduced decomposition used to define the PBW basis is not adapted to any orientation of the graph of $\mathfrak{g}$.

Received by the editors October 6, 2010 and, in revised form, August 22, 2011.

2010 Mathematics Subject Classification. Primary 05E10; Secondary 16G20, 17B10, 22E46, $52 \mathrm{~B} 20$. 
To construct the modules $N(\gamma)$ and study the functions $D_{\gamma}$, a key tool is reflection functors for modules over the preprojective algebra. These functors extend the usual BGP (Bernstein, Gelfand, Ponomarev) reflection functors [5] to the preprojective framework; they are different from Crawley-Boevey and Holland's reflection functors 8 for the deformed preprojective algebra. Our initial motivation to define them was to understand the meaning of Kashiwara and Saito's crystal reflections in terms of the semicanonical basis.

The combinatorics of MV polytopes was originally developed by Anderson and the second author, in order to describe the Mirković-Vilonen cycles in affine Grassmannians. Affine Grassmannians and quiver varieties are two geometric constructions of representations of $\mathfrak{g}$ and they each give their own basis for representations. One motivation for the current paper was to relate the geometry of affine Grassmannians and nilpotent varieties as part of a bigger project which seeks to understand and compare these bases. In particular, in this paper we have achieved a labelling of the irreducible components of nilpotent varieties by MV polytopes and hence a natural bijection between these components and MV cycles. Moreover, we have done so by constructing functions $D_{\gamma}$ which are the direct analogs of functions introduced by the second author in [13] for the study of MV cycles. In fact, the functions $D_{\gamma}$ define a stratification of the varieties $\Lambda(\nu)$. The strata are indexed by the same pseudo-Weyl polytopes that index the GGMS (Gelfand, Goresky, MacPherson, Serganova) strata of the affine Grassmannian.

Another motivation of the current project is to develop a theory of MV polytopes for affine Kac-Moody Lie algebras. From the MV cycles perspective this is difficult, since the affine Grassmannian for Kac-Moody groups is not yet available. However, the theory of quivers extends nicely to the Kac-Moody setting. In a forthcoming work [3], we will (with Peter Tingley) extend the results of this paper to quivers for affine Kac-Moody Lie algebras and obtain a notion of MV polytopes in this setting.

Geiß, Leclerc and Schröer explained to us that their work on the cluster properties of the semicanonical basis also relies on the modules $N(\gamma)$, which they introduce differently. This connection will be further explored in [3].

In a related work [14], the second author and C. Sadanand analyzed explicity the case of $\mathfrak{g}=\mathfrak{s l}_{n}$ and compared the results of the current paper with a previous description of the components of nilpotent varieties obtained by A. Savage [32]. In particular, Theorem 4.3 of [14] can be obtained from the results of this paper by applying a well-known recipe for producing semistandard Young tableaux from MV polytopes for $\mathfrak{s l}_{n}$.

\section{REFLECTION FUnCTORS}

We begin this section by reviewing the definition of the preprojective algebra $\Pi(Q)$ of a quiver $Q$. Then we define and study reflection functors for $\Pi(Q)$-modules.

2.1. Recall on preprojective algebras. Let $K$ be a field, fixed for the whole paper. Let $Q=(I, E)$ be a quiver with vertex set $I$ and arrow set $E$. We denote by $s$ and $t$ the source and target maps from $E$ to $I$. We denote the path algebra of $Q$ over $K$ by $K Q$. Then a $K Q$-module $M$ is the data of an $I$-graded vector space $\bigoplus_{i \in I} M_{i}$ and of linear maps $M_{a}: M_{s(a)} \rightarrow M_{t(a)}$ for each arrow $a \in E$.

To each arrow $a: i \rightarrow j$ in $E$, we associate an arrow $a^{*}: j \rightarrow i$ going in the opposite direction. We let $H=E \sqcup E^{*}$ and we extend $*$ to $H$ by setting $\left(a^{*}\right)^{*}=a$. 
We extend the source and target maps to $H$ and we set $\varepsilon(a)=1$ if $a \in E$ and $\varepsilon(a)=-1$ if $a \in E^{*}$.

The quotient of the path algebra of $(I, H)$ by the ideal generated by

$$
\sum_{a \in H} \varepsilon(a) a a^{*}
$$

is called the preprojective algebra of $Q$ and is denoted by $\Pi(Q)$. Thus a $\Pi(Q)$ module $M$ is the data of an $I$-graded vector space $\bigoplus_{i \in I} M_{i}$ and of linear maps $M_{a}: M_{s(a)} \rightarrow M_{t(a)}$ for each arrow $a \in H$, which satisfy

$$
\sum_{\substack{a \in H \\ t(a)=i}} \varepsilon(a) M_{a} M_{a^{*}}=0
$$

for each $i \in I$. In this paper, we will only consider finite-dimensional modules.

Example 2.1. When $Q$ has no loops nor multiple arrows, one may depict $\Pi(Q)$ modules in a simple way, by using a symbol $i$ to represent vectors from a basis for $M_{i}$ and by drawing arrows to indicate the action of the linear maps. For instance, let $Q$ be the quiver

$$
1 \stackrel{a}{\leftarrow} 2 \stackrel{b}{\rightarrow} 3
$$

of type $A_{3}$. The $\Pi(Q)$-module $M$ represented by the diagram

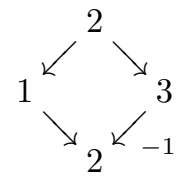

has dimension-vector $\left(\operatorname{dim} M_{1}, \operatorname{dim} M_{2}, \operatorname{dim} M_{3}\right)=(1,2,1)$; the map $M_{a}$ sends the top basis vector in $M_{2}$ to the basis vector in $M_{1}$ and the map $M_{b^{*}}$ sends the basis vector in $M_{3}$ to the negative of the bottom basis vector in $M_{2}$.

The dual of a $\Pi(Q)$-module $M$ is the $\Pi(Q)$-module $M^{*}$ defined by taking the dual spaces and maps as follows:

$$
\left(M^{*}\right)_{i}=\left(M_{i}\right)^{*} \text { and }\left(M^{*}\right)_{a}=\left(M_{a^{*}}\right)^{*}
$$

for all $i \in I$ and $a \in H$. This duality is an involutive antiautoequivalence $*$ on the category of $\Pi(Q)$-modules.

To any $\Pi(Q)$-module $M$, one associates the tuple $\underline{\operatorname{dim}} M=\left(\operatorname{dim} M_{i}\right)$ in $\mathbb{N}^{I}$, called the dimension-vector of $M$. Conversely, given a dimension-vector $\nu \in \mathbb{N}^{I}$, we set $M_{i}=K^{\nu_{i}}$ and denote by $\Lambda(\nu)$ the variety of all matrices

$$
\left(M_{a}\right) \in \prod_{a \in H} \operatorname{Hom}_{K}\left(M_{s(a)}, M_{t(a)}\right)
$$

that satisfy the preprojective relations

$$
\sum_{\substack{a \in H \\ t(a)=i}} \varepsilon(a) M_{a} M_{a^{*}}=0
$$

at each vertex $i \in I$. Thus a point in $\Lambda(\nu)$ is a representation of $\Pi(Q)$ on the vector space $\bigoplus_{i \in I} M_{i}$. The group

$$
G(\nu)=\prod_{i \in I} \operatorname{Aut}\left(M_{i}\right)
$$


acts on $\Lambda(\nu)$ by conjugation. The orbits of this action are in canonical bijection with the isomorphism classes of $\Pi(Q)$-modules with dimension-vector $\nu$.

The lattice $\mathbb{Z}^{I}$ is equipped with a symmetric bilinear form defined by

$$
\langle\mu, \nu\rangle=2 \sum_{i \in I} \mu_{i} \nu_{i}-\sum_{a \in H} \nu_{s(a)} \mu_{t(a)} .
$$

The following formula is due to Crawley-Boevey ([7, Lemma 1): if $M$ and $N$ are two $\Pi(Q)$-modules, then

$\langle\underline{\operatorname{dim}} M, \underline{\operatorname{dim}} N\rangle=\operatorname{dim} \operatorname{Hom}_{\Pi(Q)}(M, N)+\operatorname{dim} \operatorname{Hom}_{\Pi(Q)}(N, M)-\operatorname{dim}_{\operatorname{Ext}_{\Pi(Q)}^{1}}^{1}(M, N)$.

We denote the standard basis of $\mathbb{Z}^{I}$ by $\left(\alpha_{i}\right)$. Given a vertex $i$, we denote by $S_{i}$ the $\Pi(Q)$-module of dimension one concentrated at $i$ on which all arrows act as zero; thus $\underline{\operatorname{dim}} S_{i}=\alpha_{i}$. Given a $\Pi(Q)$-module $M$, the $i$-socle of $M$, denoted $\operatorname{soc}_{i} M$, is the $S_{i}$-isotypic component of the socle of $M$; likewise, the $i$-head of $M$, denoted $\operatorname{hd}_{i} M$, is the $S_{i}$-isotypic component of the head of $M$. Thus $\operatorname{soc}_{i}$ and hd are endofunctors on the category of $\Pi(Q)$-modules.

Remark 2.2. Usually, one restricts attention to representations that satisfies a nilpotency condition (see 19, section 8.2). This amounts to investigating the full subcategory of $\Pi(Q)$-mod formed by the modules whose composition factors all belong to the set $\left\{S_{i} \mid i \in I\right\}$. It can, however, be shown that these nilpotency conditions are automatically satisfied in the case where $Q$ is of type $A D E$ (see Satz 1 in [26] or Proposition 14.2 (a) in 20]). In this case, $\left\{S_{i} \mid i \in I\right\}$ is a complete set of simple $\Pi(Q)$-modules, and thus a $\Pi(Q)$-module with trivial $i$-socle for all $i \in I$ is itself trivial.

2.2. Definition of the reflection functors. We fix a vertex $i \in I$ at which $Q$ has no loops. Our aim is to define a pair of adjoint endofunctors $\left(\Sigma_{i}^{*}, \Sigma_{i}\right)$ on the category of $\Pi(Q)$-modules.

We break the datum of a $\Pi(Q)$-module $M$ into two parts. The first part consists of the vector spaces $M_{j}$ for $j \neq i$ and of the linear maps between them; the second part consists of the vector spaces and of the linear maps that appear in the diagram

$$
\bigoplus_{\substack{a \in H \\ t(a)=i}} M_{s(a)} \stackrel{\left(\varepsilon(a) M_{a}\right)}{\longrightarrow} M_{i} \stackrel{\left(M_{a^{*}}\right)}{\longrightarrow} \bigoplus_{\substack{a \in H \\ t(a)=i}} M_{s(a)} .
$$

For brevity, we will write this diagram as

$$
\widetilde{M}_{i} \stackrel{M_{\mathrm{in}(i)}}{\longrightarrow} M_{i} \stackrel{M_{\mathrm{out}(i)}}{\longrightarrow} \widetilde{M}_{i} .
$$

The preprojective relation at $i$ is $M_{\mathrm{in}(i)} M_{\text {out }(i)}=0$.

We now construct a new $\Pi(Q)$-module by replacing $(*)$ with

$$
\widetilde{M}_{i} \stackrel{\bar{M}_{\text {out }(i)} M_{\mathrm{in}(i)}}{\longrightarrow} \operatorname{ker} M_{\mathrm{in}(i)} \hookrightarrow \widetilde{M}_{i},
$$

where the map $\bar{M}_{\text {out }(i)}: M_{i} \rightarrow \operatorname{ker} M_{\text {in }(i)}$ is induced by $M_{\text {out }(i)}$. We glue this new datum with the remaining part of $M$. The preprojective relations are still satisfied, because the replacement does not change the endomorphism $M_{\text {out }(i)} M_{\operatorname{in}(i)}$ of $\widetilde{M}_{i}$. We thus end up with a new $\Pi(Q)$-module, which we denote by $\Sigma_{i} M$.

Likewise, we may replace $(*)$ with

$$
\widetilde{M}_{i} \rightarrow \operatorname{coker} M_{\mathrm{out}(i)} \stackrel{M_{\mathrm{out}(i)} \bar{M}_{\mathrm{in}(i)}}{\longrightarrow} \widetilde{M}_{i},
$$


where the map $\bar{M}_{\text {in }(i)}:$ coker $M_{\text {out }(i)} \rightarrow M_{i}$ is induced by $M_{\text {in }(i)}$. We again end up with a new $\Pi(Q)$-module, which we denote by $\Sigma_{i}^{*} M$.

One checks without difficulty that these constructions define covariant additive functors $\Sigma_{i}$ and $\Sigma_{i}^{*}$, that $\Sigma_{i}$ is left-exact and that $\Sigma_{i}^{*}$ is right-exact.

Example 2.3. Let $Q$ be a quiver of type $A_{3}$. The diagram below presents the action of $\Sigma_{2}$ and $\Sigma_{2}^{*}$ on several $\Pi(Q)$-modules.

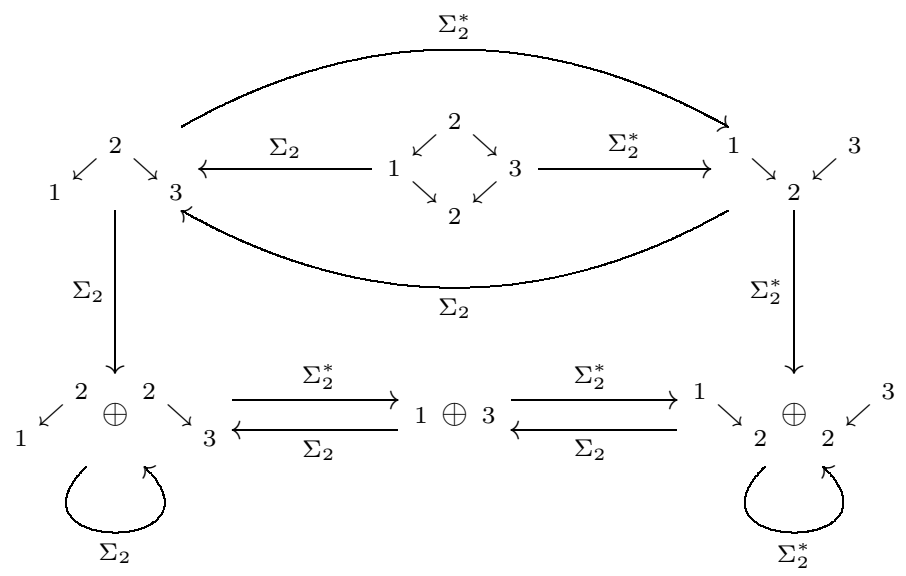

Remark 2.4. (i) We could as well distribute the signs $\varepsilon(a)$ differently in (*), but that would just change $\Sigma_{i}$ by an isomorphism. Indeed, write $M_{\mathrm{in}(i)}^{\prime}$ and $M_{\text {out }(i)}^{\prime}$ for the two maps that appear in

$$
\bigoplus_{\substack{a \in H \\ t(a)=i}} M_{s(a)} \stackrel{\left(M_{a}\right)}{\longrightarrow} M_{i} \stackrel{\left(\varepsilon(a) M_{a^{*}}\right)}{\longrightarrow} \bigoplus_{\substack{a \in H \\ t(a)=i}} M_{s(a)}
$$

Then the module $\Sigma_{i} M$ defined above fits in a commutative diagram

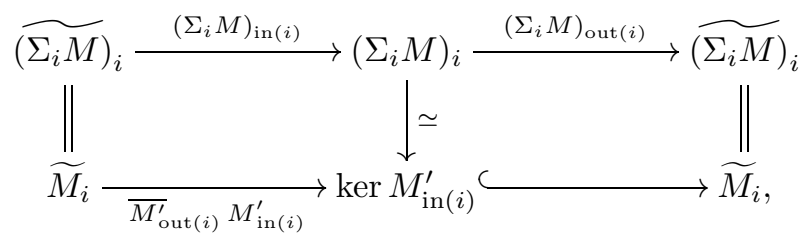

where the middle vertical arrow is the map $\left(x_{a}\right) \mapsto\left(\varepsilon(a) x_{a}\right)$, both spaces $\left(\Sigma_{i} M\right)_{i}$ and $\operatorname{ker} M_{\mathrm{in}(i)}^{\prime}$ being viewed as subspaces of

$$
\widetilde{M}_{i}=\bigoplus_{\substack{a \in H \\ t(a)=i}} M_{s(a)}
$$

A similar remark can be stated about $\Sigma_{i}^{*}$. As a consequence, we see that $\Sigma_{i}^{*} \cong * \Sigma_{i} *$. 
(ii) The commutative diagram

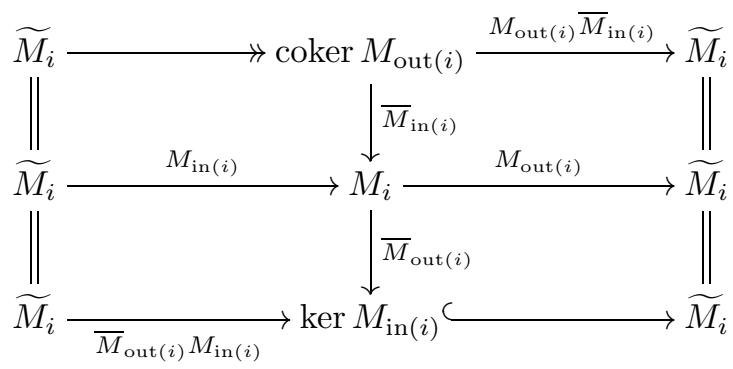

shows the existence of canonical morphisms $\Sigma_{i}^{*} M \rightarrow M \rightarrow \Sigma_{i} M$.

(iii) Let $I_{i}$ be the annihilator of the module $S_{i}$. This two-sided ideal in $\Pi(Q)$ has been studied at length by Iyama, Reiten et al.; see for instance 6. It allows us to represent our reflection functors as $\Sigma_{i}=\operatorname{Hom}_{\Pi(Q)}\left(I_{i}, ?\right)$ and $\Sigma_{i}^{*}=I_{i} \otimes_{\Pi(Q)}$ ?. We owe this observation to Amiot; the proof will be given in our forthcoming paper 3 . In view of this fact, our functors $\Sigma_{i}$ and $\Sigma_{i}^{*}$ are not new.

\section{Proposition 2.5. $\quad$ (i) The pair $\left(\Sigma_{i}^{*}, \Sigma_{i}\right)$ is a pair of adjoint functors.}

(ii) The adjunction morphisms id $\rightarrow \Sigma_{i} \Sigma_{i}^{*}$ and $\Sigma_{i}^{*} \Sigma_{i} \rightarrow$ id can be inserted in functorial short exact sequences

$$
0 \rightarrow \operatorname{soc}_{i} \rightarrow \mathrm{id} \rightarrow \Sigma_{i} \Sigma_{i}^{*} \rightarrow 0 \quad \text { and } \quad 0 \rightarrow \Sigma_{i}^{*} \Sigma_{i} \rightarrow \mathrm{id} \rightarrow \mathrm{hd}_{i} \rightarrow 0 .
$$

Proposition 2.5 (ii) readily implies that $\Sigma_{i}$ and $\Sigma_{i}^{*}$ define inverse equivalences of categories

$$
\left\{\begin{array}{c}
\Pi(Q) \text {-modules } \\
\text { with trivial } i \text {-head }
\end{array}\right\} \stackrel{\Sigma_{i}}{\longleftarrow}\left\{\begin{array}{c}
\Pi(Q) \text {-modules } \\
\text { with trivial } i \text {-socle }
\end{array}\right\}
$$

and that there are natural isomorphisms

$$
\Sigma_{i} \Sigma_{i}^{*} \Sigma_{i} \cong \Sigma_{i} \text { and } \Sigma_{i}^{*} \Sigma_{i} \Sigma_{i}^{*} \cong \Sigma_{i}^{*} .
$$

Let $s_{i}$ be the reflection $\mu \mapsto \mu-\left\langle\alpha_{i}, \mu\right\rangle \alpha_{i}$ on the lattice $\mathbb{Z}^{I}$. Routine arguments show that

$$
\operatorname{hd}_{i} M=0 \Longrightarrow \underline{\operatorname{dim}} \Sigma_{i} M=s_{i}(\underline{\operatorname{dim}} M) .
$$

We leave as an exercise for the reader to prove that

$$
\operatorname{hd}_{i} M=0 \Longleftrightarrow \underline{\operatorname{dim}} \Sigma_{i} M=s_{i}(\underline{\operatorname{dim}} M) \Longleftrightarrow M \cong \Sigma_{i}^{*} \Sigma_{i} M
$$

and

$$
\operatorname{soc}_{i} M=0 \Longleftrightarrow \underline{\operatorname{dim}} \Sigma_{i}^{*} M=s_{i}(\underline{\operatorname{dim}} M) \Longleftrightarrow M \cong \Sigma_{i} \Sigma_{i}^{*} M ;
$$

we will, however, not use this more complete result.

Proof. To establish (i) it is enough to define a pair of converse bijections

$$
\operatorname{Hom}_{\Pi(Q)}\left(M, \Sigma_{i} N\right) \cong \operatorname{Hom}_{\Pi(Q)}\left(\Sigma_{i}^{*} M, N\right)
$$

for any modules $M$ and $N$, which are natural in $M$ and $N$. The construction is as follows. 
Consider a morphism $f: M \rightarrow \Sigma_{i} N$. By definition, this is a collection of linear maps $f_{j}: M_{j} \rightarrow\left(\Sigma_{i} N\right)_{j}$, for all $j \in I$, which intertwine the action of the arrows in $H$. Set

$$
\widetilde{f}_{i}=\bigoplus_{\substack{a \in H \\ t(a)=i}} f_{s(a)}: \widetilde{M}_{i} \rightarrow \widetilde{N}_{i}
$$

In the diagram

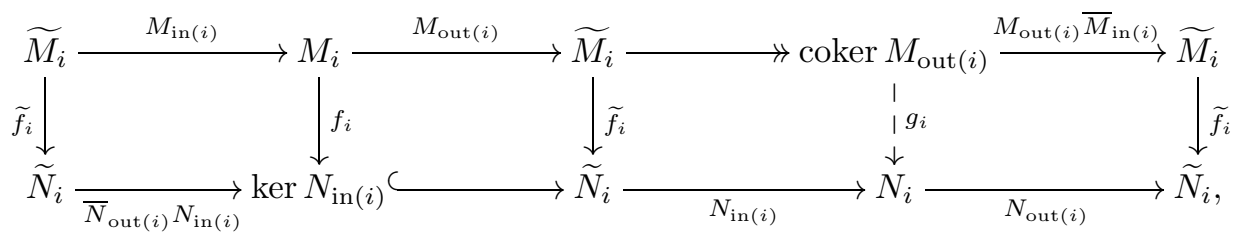

the two left squares commute. There is thus a unique map $g_{i}$ making the third square commutative. The fourth square then also commutes, so that if we set $g_{j}=f_{j}$ for all the vertices $j \neq i$, we get a morphism $g: \Sigma_{i}^{*} M \rightarrow N$.

Conversely, consider a morphism $g: \Sigma_{i}^{*} M \rightarrow N$ and set

$$
\widetilde{g}_{i}=\bigoplus_{\substack{a \in H \\ t(a)=i}} g_{s(a)}: \widetilde{M}_{i} \rightarrow \widetilde{N}_{i} .
$$

In the diagram

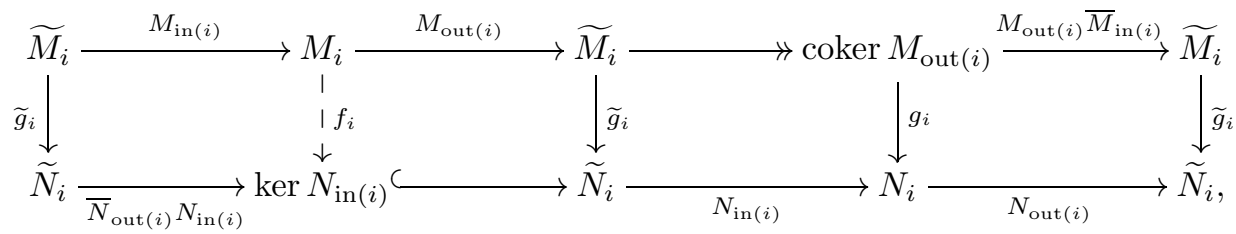

the two right squares commute. There is thus a unique map $f_{i}$ making the second square commutative. The first square then also commutes, so that if we set $f_{j}=g_{j}$ for all the vertices $j \neq i$, we get a morphism $f: M \rightarrow \Sigma_{i} N$.

To establish (ii)] one checks that $\Sigma_{i}^{*} \Sigma_{i} M$ is the $\Pi(Q)$-module obtained by replacing in $M$ the part summed up by $(*)$ with

$$
\widetilde{M}_{i} \stackrel{M_{\mathrm{in}(i)}}{\longrightarrow} \operatorname{im} M_{\mathrm{in}(i)} \stackrel{M_{\mathrm{out}(i)}}{\longrightarrow} \widetilde{M}_{i}
$$

and that $\Sigma_{i} \Sigma_{i}^{*} M$ is the $\Pi(Q)$-module obtained by replacing in $M$ the part summed up by $(*)$ with

$$
\widetilde{M}_{i} \stackrel{M_{\text {out }(i)} M_{\text {in }(i)}}{\longrightarrow} \operatorname{im} M_{\text {out }(i)} \hookrightarrow \widetilde{M}_{i} .
$$

It remains to observe that as vector spaces, $\operatorname{hd}_{i} M \cong \operatorname{coker} M_{\mathrm{in}(i)}$ and $\operatorname{soc}_{i} M \cong$ $\operatorname{ker} M_{\text {out }(i)}$.

2.3. Further properties. We begin this section with an easy lemma, which is used in the proof of Proposition 3.5.

Lemma 2.6. If the $\Pi(Q)$-module $M$ has trivial $i$-socle, then the canonical morphism $M \rightarrow \Sigma_{i} M$ is a monomorphism which induces isomorphisms $\Sigma_{i} M \cong \Sigma_{i}^{2} M$ and $\operatorname{soc} M \cong \operatorname{soc}\left(\Sigma_{i} M\right)$. 
Proof. The $i$-socle of $M$ is the kernel of the map $M_{\text {out }(i)}$. Assume that it is trivial. Then $\bar{M}_{\text {out }(i)}$ is injective. The canonical morphism $f: M \rightarrow \Sigma_{i} M$ is thus a monomorphism, and the equality $\operatorname{ker} M_{\mathrm{in}(i)}=\operatorname{ker}\left(\bar{M}_{\mathrm{out}(i)} M_{\mathrm{in}(i)}\right)$ holds, which implies $\Sigma_{i} M \cong \Sigma_{i}^{2} M$.

The module soc $\Sigma_{i} M$ cannot map non-trivially to the cokernel of $f$, for the latter is a direct sum of copies of $S_{i}$ while $\Sigma_{i} M$ has trivial $i$-socle. Applying the functor $\operatorname{Hom}_{\Pi(Q)}\left(\operatorname{soc} \Sigma_{i} M, ?\right)$ to the short exact sequence

$$
0 \rightarrow M \stackrel{f}{\rightarrow} \Sigma_{i} M \rightarrow \text { coker } f \rightarrow 0
$$

we deduce that the canonical inclusion $\operatorname{soc}\left(\Sigma_{i} M\right) \hookrightarrow \Sigma_{i} M$ factorizes through $f$. Therefore $f$ maps soc $M$ onto $\operatorname{soc}\left(\Sigma_{i} M\right)$.

In 8], Crawley-Boevey and Holland define reflection functors on the category of modules over the deformed preprojective algebra. Their functors define an action of the Weyl group. Our functors do not enjoy the property $\Sigma_{i}^{2}=$ id, but it is nevertheless reassuring to see that they satisfy the braid relations.

Proposition 2.7. Let $i$ and $j$ be two vertices that are linked by a single edge in $Q$. Then the functors $\Sigma_{i} \Sigma_{j} \Sigma_{i}$ and $\Sigma_{j} \Sigma_{i} \Sigma_{j}$ are isomorphic.

Proof. We only sketch the proof, since we will not use this property later on. Let $\left(c, c^{*}\right)$ be the pair of arrows in $H$ between the vertices $i$ and $j$. Let $M$ be a $\Pi(Q)$ module. Abbreviate the part

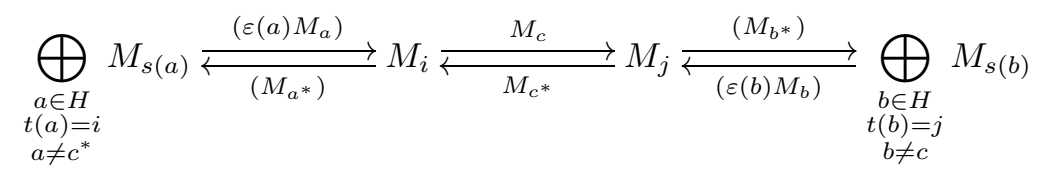

of the datum of $M$ by the notation

$$
R \underset{l}{\stackrel{k}{\rightleftarrows}} V \underset{g}{\stackrel{f}{\rightleftarrows}} W \underset{n}{\stackrel{m}{\rightleftarrows}} S
$$

The preprojective relations at $i$ and $j$ then read

$$
k l+\varepsilon\left(c^{*}\right) g f=0 \quad \text { and } \quad n m+\varepsilon(c) f g=0 .
$$

Explicit computations show that applying $\Sigma_{i} \Sigma_{j} \Sigma_{i}$ or $\Sigma_{j} \Sigma_{i} \Sigma_{j}$ to $M$ both amount to replacing this part of the datum of $M$ with

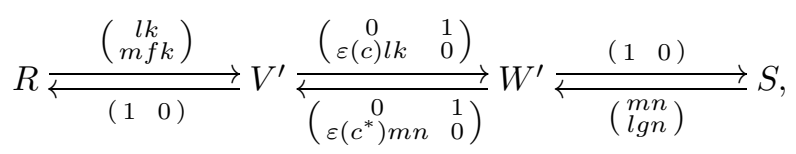

where $V^{\prime} \subseteq R \oplus S$ and $W^{\prime} \subseteq S \oplus R$ are the kernels of the linear maps

$$
R \oplus S \stackrel{(f k \quad n)}{\longrightarrow} W \quad \text { and } \quad S \oplus R \stackrel{(g n k)}{\longrightarrow} V .
$$




\section{The Modules $N(\gamma)$}

From now on, we fix a Dynkin diagram $\Gamma$ of type $A D E$. Orienting the edges of this diagram yields a quiver $Q=(I, E)$. The datum of the diagram $\Gamma$ is equivalent to that of the isomorphism class of a simply-laced semisimple complex Lie algebra $\mathfrak{g}$. The set $I$ of vertices of $\Gamma$ indexes the simple roots $\alpha_{i}$ of $\mathfrak{g}$. The root lattice $Q$ is then the lattice $\mathbb{Z}^{I}$ with basis $\left(\alpha_{i}\right)_{i \in I}$; we write $Q_{+}$for the image of $\mathbb{N}^{I}$ in this identification. (We are here using the same notation $Q$ for the quiver and for the root lattice. These notations are traditional and we are convinced that our choice will not be a source of confusion for the reader.) Since we are in the simply laced case, there is no need to distinguish between roots and coroots. The weight lattice is thus the dual $P$ of this lattice $Q$; it is endowed with the basis $\left(\omega_{i}\right)_{i \in I}$ of fundamental weights. By duality, the reflections $s_{i}$ defined at the end of section 2.2 act on $P$ and generate the Weyl group $W \subseteq \operatorname{Aut}(P)$. Then $\left(W,\left(s_{i}\right)_{i \in I}\right)$ is a finite Coxeter system; we denote its length function by $\ell$ and its longest element by $w_{0}$. Finally, the bilinear form $\langle$,$\rangle from section 2.1$ is the standardly normalised $W$-invariant scalar product on $Q$; it embeds $Q$ as a sublattice of $P$.

Our aim in this section is to define a family of $\Pi(Q)$-modules $N(\gamma)$ indexed by weights $\gamma \in P$. These modules can be characterized up to isomorphism in the following fashion.

Theorem 3.1. $\quad$ (i) If $\gamma$ is antidominant, then $N(\gamma)=0$.

(ii) Let $i \in I$ and let $\gamma$ be a $W$-conjugate of $-\omega_{i}$, with $\gamma \neq-\omega_{i}$. Then $N(\gamma)$ satisfies

$$
\underline{\operatorname{dim}} N(\gamma)=\gamma+\omega_{i} \quad \text { and } \quad \operatorname{soc} N(\gamma) \cong S_{i} .
$$

Moreover, these conditions characterize $N(\gamma)$ up to isomorphism.

(iii) If $\gamma$ and $\delta$ belong to the same Weyl chamber, then $N(\gamma+\delta) \cong N(\gamma) \oplus N(\delta)$.

This theorem will be proved in section 3.4.

3.1. Nakajima's quiver. The easiest way to define the modules $N(\gamma)$ uses Nakajima's trick (see [24]) of expanding $Q$ by adding an extra vertex $i^{\prime}$ and an extra arrow $d_{i}: i \rightarrow i^{\prime}$ for each vertex $i \in I$. Playing the same game as in section 2.1, we double all arrows in this extended quiver, obtaining thereby a set of arrows $\widehat{H}=H \sqcup\left\{d_{i}, d_{i}^{*} \mid i \in I\right\}$ with an involution *. Here is an example depicting the situation for the type $A_{3}$.

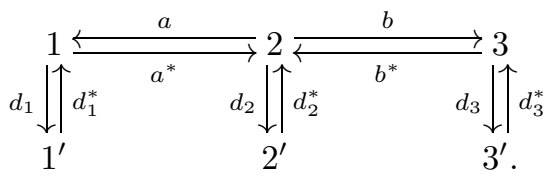

We then construct the preprojective algebra of the extended quiver, which we denote by $\Pi(\widehat{Q})$. Hence a $\Pi(\widehat{Q})$-module consists of two $I$-graded vector spaces $\bigoplus_{i \in I} M_{i}$ and $\bigoplus_{i \in I} M_{i^{\prime}}$ along with linear maps $M_{a}: M_{s(a)} \rightarrow M_{t(a)}$, for $a \in H$, and $M_{d_{i}}: M_{i} \rightarrow M_{i^{\prime}}$ and $M_{d_{i}^{*}}: M_{i^{\prime}} \rightarrow M_{i}$, for $i \in I$, which satisfy the preprojective relations

$$
\sum_{\substack{a \in H \\ t(a)=i}} \varepsilon(a) M_{a} M_{a^{*}}=M_{d_{i}^{*}} M_{d_{i}} \quad \text { and } \quad M_{d_{i}} M_{d_{i}^{*}}=0
$$


at each vertex $i \in I$. The dimension-vector of $M$ is defined as the pair $\underline{\operatorname{dim}} M=$ $(\nu, \lambda)$ in $\mathbb{N}^{I} \times \mathbb{N}^{I^{\prime}}$, where $\nu_{i}=\operatorname{dim} M_{i}$ and $\lambda_{i^{\prime}}=\operatorname{dim} M_{i^{\prime}}$.

Given a dimension-vector $(\nu, \lambda) \in \mathbb{N}^{I} \times \mathbb{N}^{I^{\prime}}$ for $\Pi(\widehat{Q})$, it is customary to view $\lambda=\left(\lambda_{i^{\prime}}\right)$ as the antidominant weight $-\sum_{i \in I} \lambda_{i^{\prime}} \omega_{i}$ in $P$. The main justification for this identification is that the pairing between $P$ and $Q$ coincides with the restriction to $\mathbb{Z}^{I^{\prime}}$ and $\mathbb{Z}^{I}$ of the symmetric bilinear form of the extended quiver. This fact will be exploited below when we write expressions like

$$
\left\langle(\nu, \lambda),\left(\alpha_{i}, 0\right)\right\rangle=\left\langle\nu+\lambda, \alpha_{i}\right\rangle ;
$$

here the left-hand side is the symmetric bilinear form from section 2.1 and the right-hand side is the duality pairing between $P$ and $Q$.

3.2. Stable $\Pi(\widehat{Q})$-modules. We say that a $\Pi(\widehat{Q})$-module $M$ is stable if for each $i \in I$, the linear map

$$
M_{i} \stackrel{\left(\begin{array}{c}
\left(M_{a}\right) \\
M_{d_{i}}
\end{array}\right)}{\longrightarrow}\left(\bigoplus_{\substack{a \in H \\
s(a)=i}} M_{t(a)}\right) \oplus M_{i^{\prime}}
$$

is injective. In other words, we ask that $\operatorname{soc}_{i} M=0$ at each non-primed vertex.

This notion of stability was introduced by Nakajima. Indeed, using Remark 2.2 one can easily see that this definition is equivalent to Definition 3.9 in 25. We now study the stable $\Pi(\widehat{Q})$-modules following ideas of Nakajima and Saito.

Lemma 3.2. Let $M$ be a stable $\Pi(\widehat{Q})$-module with dimension-vector $(\nu, \lambda)$. Let $c$ be the dimension of the $i$-head of $M$. Then

$$
\operatorname{dim} \operatorname{Hom}_{\Pi(\widehat{Q})}\left(M, S_{i}\right)=c \quad \text { and } \quad \operatorname{dim}_{\operatorname{Ext}_{\Pi(\widehat{Q})}^{1}}\left(M, S_{i}\right)=c-\left\langle\nu+\lambda, \alpha_{i}\right\rangle .
$$

In particular, $c \geq \max \left(0,\left\langle\nu+\lambda, \alpha_{i}\right\rangle\right)$.

Proof. The first equality is equivalent to the assertion $\operatorname{dim} \operatorname{hd}_{i} M=c$. On the other hand, the stability condition means that $\operatorname{Hom}_{\Pi(\widehat{Q})}\left(S_{i}, M\right)=0$. Now the dimensionvector of $S_{i}$ is $\left(\alpha_{i}, 0\right)$, hence the result follows from Crawley-Boevey's formula (see section 2.1).

Let $M$ be a stable $\Pi(\widehat{Q})$-module with dimension-vector $(\nu, \lambda)$. By Lemma 3.2 , the $i$-head of $M$ has dimension at least $\max \left(0,\left\langle\nu+\lambda, \alpha_{i}\right\rangle\right)$. If there is equality here, then we say that $M$ has a small $i$-head.

Lemma 3.3. $\quad$ (i) Let $M$ be a stable $\Pi(\widehat{Q})$-module with dimension-vector $(\nu, \lambda)$ whose $i$-head has dimension $c$. Then for any integer $k$ such that $-c \leq k \leq$ $c-\left\langle\nu+\lambda, \alpha_{i}\right\rangle$, there exists a stable $\Pi(\widehat{Q})$-module with dimension-vector $\left(\nu+k \alpha_{i}, \lambda\right)$.

(ii) Assume that $\left\langle\nu+\lambda, \alpha_{i}\right\rangle \leq 0$. Then the functors $\Sigma_{i}$ and $\Sigma_{i}^{*}$ restrict to equivalences of categories:

$\left\{\begin{array}{c}\text { stable } \Pi(\widehat{Q}) \text {-modules with } \\ \text { dimension-vector }(\nu, \lambda) \\ \text { with small } i \text {-head }\end{array}\right\} \stackrel{\Sigma_{i}}{\longleftarrow}\left\{\begin{array}{c}\text { stable } \Pi(\widehat{Q}) \text {-modules with } \\ \text { dimension-vector }\left(\nu-\left\langle\nu+\lambda, \alpha_{i}\right\rangle \alpha_{i}, \lambda\right) \\ \text { with small } i \text {-head }\end{array}\right\}$. 
(iii) If there exists a stable $\Pi(\widehat{Q})$-module with dimension-vector $(\nu, \lambda)$, then

$$
\nu+\lambda \in \bigcap_{w \in W} w\left(\lambda+Q_{+}\right)
$$

Proof. Items (i) and (ii) are Lemma 4.2.1 and a special case of Lemma 4.2.2 in 30. Item (iii) is a consequence of Corollary 10.8 in [25]. We, nevertheless, recall the proof of all these facts for the convenience of the reader.

Let $M$ be a stable $\Pi(\widehat{Q})$-module with dimension-vector $(\nu, \lambda)$. We analyze the situation locally around the vertex $i$. For brevity, let us introduce the notation $\widetilde{M}_{i}$, $M_{\mathrm{in}(i)}$ and $M_{\mathrm{out}(i)}$ for the objects in the diagram

$$
\left(\bigoplus_{\substack{a \in H \\
t(a)=i}} M_{s(a)}\right) \oplus M_{i^{\prime}} \stackrel{\left(\left(\varepsilon(a) M_{a}\right)-M_{d_{i}^{*}}\right)}{\longrightarrow} M_{i} \stackrel{\left(\begin{array}{c}
\left(M_{a^{*}}\right) \\
M_{d_{i}}
\end{array}\right)}{\longrightarrow}\left(\bigoplus_{\substack{a \in H \\
t(a)=i}} M_{s(a)}\right) \oplus M_{i^{\prime}}
$$

as we did in section 2.2. The stability condition means that $M_{\text {out }(i)}$ is injective.

Consider the endomorphism $u=M_{\mathrm{out}(i)} M_{\mathrm{in}(i)}$ of $\widetilde{M}_{i}$. Note that

$$
\operatorname{im} u \subseteq \operatorname{im} M_{\text {out }(i)} \subseteq \operatorname{ker} u,
$$

that

and that

$$
\operatorname{dimim} M_{\text {out }(i)}-\operatorname{dimim} u=\operatorname{dim} \operatorname{coker} M_{\operatorname{in}(i)}=c,
$$

$$
\begin{aligned}
\operatorname{dim} \operatorname{ker} u-\operatorname{dimim} M_{\text {out }(i)} & =\operatorname{dim} \widetilde{M}_{i}-\operatorname{dim} \operatorname{im} u-\operatorname{dim} \operatorname{im} M_{\text {out }(i)} \\
& =c+\operatorname{dim} \widetilde{M}_{i}-2 \operatorname{dim} M_{i} \\
& =c-\left\langle\nu+\lambda, \alpha_{i}\right\rangle .
\end{aligned}
$$

Thus for any $k$ satisfying $-c \leq k \leq c-\left\langle\nu+\lambda, \alpha_{i}\right\rangle$, we can find a subspace $V \subseteq \widetilde{M}_{i}$ of dimension $k+\operatorname{dim} M_{i}$ satisfying $\operatorname{im} u \subseteq V \subseteq \operatorname{ker} u$. We can then construct a new $\Pi(\widehat{Q})$-module by replacing

$$
\widetilde{M}_{i} \stackrel{M_{\mathrm{in}(i)}}{\longrightarrow} M_{i} \stackrel{M_{\mathrm{out}(i)}}{\longrightarrow} \widetilde{M}_{i} \quad \text { with } \quad \widetilde{M}_{i} \stackrel{u}{\longrightarrow} V \hookrightarrow \widetilde{M}_{i}
$$

in $M$. We thus get a stable $\Pi(\widehat{Q})$-module of dimension-vector $\left(\nu+k \alpha_{i}, \lambda\right)$, which establishes (i).

Keeping the same notation, we now observe that $M$ has a small $i$-head if and only if either $c$ or $c-\left\langle\nu+\lambda, \alpha_{i}\right\rangle$ equals zero. In other words, $M$ has a small $i$-head if and only if $\operatorname{im} M_{\text {out }(i)}$ is either $\operatorname{im} u$ or ker $u$. Hence keeping $\widetilde{M}_{i}$ and $u$ the same and flipping $M_{i}$ between $\operatorname{im} u$ and ker $u$ yields equivalences. This flipping is indeed what the functors $\Sigma_{i}$ and $\Sigma_{i}^{*}$ do: under the assumption $\left\langle\nu+\lambda, \alpha_{i}\right\rangle \leq 0$, if $M$ has dimension-vector $(\nu, \lambda)$, then we deal with the first possibility $\operatorname{im} M_{\operatorname{out}(i)}=\operatorname{im} u$, and $\Sigma_{i} M$ is the same module except with $M_{i}$ replaced by $\operatorname{ker} M_{\operatorname{in}(i)}=\operatorname{ker} u$; and if $M$ has dimension-vector $\left(\nu-\left\langle\nu+\lambda, \alpha_{i}\right\rangle \alpha_{i}, \lambda\right)$, then we deal with the second possibility $\operatorname{im} M_{\text {out }(i)}=\operatorname{ker} u$, and then $\Sigma_{i}^{*} M$ is the same module, except with $M_{i}$ replaced by coker $M_{\text {out }(i)}=\widetilde{M}_{i} / \operatorname{ker} u \cong \operatorname{im} u$. We have thus established (ii).

Looking now at (iii) we fix $\lambda$ and allow $\nu$ to vary. Assertion (i) implies that the set

$$
\{\nu+\lambda \in P \mid \text { there exists a stable } \Pi(\widehat{Q}) \text {-module with dimension-vector }(\nu, \lambda)\}
$$


is $W$-invariant. Since it is a subset of $\lambda+Q_{+}$, it is contained in $\bigcap_{w \in W} w\left(\lambda+Q_{+}\right)$. This establishes (iii)

3.3. The modules $\widehat{N}(\gamma)$. Lemma 3.3 allows us to quickly prove the following theorem, which can be seen as a corollary to the work of Nakajima [25] and Lusztig [22].

Theorem 3.4. Let $\lambda$ be an antidominant weight and let $w \in W$. Then there exists a unique (up to isomorphism) stable $\Pi(\widehat{Q})$-module with dimension-vector $(w \lambda-\lambda, \lambda)$. This module has a small $i$-head, for all $i \in I$.

Proof. Let $\lambda$ and $w$ be as in the statement of the theorem.

We first prove the second assertion. Let $M$ be a stable $\Pi(\widehat{Q})$-module $M$ with dimension-vector $(w \lambda-\lambda, \lambda)$ and let $i \in I$. We set $c=\operatorname{dim}_{i} M$ and distinguish two cases.

If $\ell\left(s_{i} w\right)>\ell(w)$, then we take $k=-c$ in Lemma 3.3 (i) and get the existence of a stable $\Pi(\widehat{Q})$-module with dimension-vector $\left(w \lambda-\lambda-c \alpha_{i}, \lambda\right)$. Lemma 3.3 (iii) then says that $w \lambda-c \alpha_{i} \in w\left(\lambda+Q_{+}\right)$, and therefore $-c w^{-1}\left(\alpha_{i}\right) \in Q_{+}$. Since $w^{-1}\left(\alpha_{i}\right)$ is a positive root here, we conclude that $c=0$. Moreover, $\left\langle w \lambda, \alpha_{i}\right\rangle \leq 0$ by the antidominance of $\lambda$.

If $\ell\left(s_{i} w\right)<\ell(w)$, then we take $k=c-\left\langle w \lambda, \alpha_{i}\right\rangle$ in Lemma 3.3 (i) and get the existence of a stable $\Pi(\widehat{Q})$-module with dimension-vector $\left(w \lambda-\lambda+k \alpha_{i}, \lambda\right)$. Lemma 3.3 (iii) then says that $w \lambda+k \alpha_{i} \in w\left(\lambda+Q_{+}\right)$. Since $w^{-1}\left(\alpha_{i}\right)$ is a negative root here, we obtain $k \leq 0$; but also $k \geq 0$, by Lemma 3.2, and therefore $c=$ $\left\langle w \lambda, \alpha_{i}\right\rangle$.

So in both cases, $c=\max \left(0,\left\langle w \lambda, \alpha_{i}\right\rangle\right)$, as claimed.

We now turn to the existence and uniqueness. The result is obvious for $w=1$. If $w \neq 1$, we pick $i \in I$ such that $\ell\left(s_{i} w\right)<\ell(w)$. Then Lemma 3.3 (ii) ensures that

$$
\left\{\begin{array}{c}
\text { stable } \Pi(\widehat{Q}) \text {-modules with } \\
\text { dimension-vector }\left(s_{i} w \lambda-\lambda, \lambda\right)
\end{array}\right\} \stackrel{\Sigma_{i}}{\longleftarrow}\left\{\begin{array}{c}
\text { stable } \Pi(\widehat{Q}) \text {-modules with } \\
\text { dimension-vector }(w \lambda-\lambda, \lambda)
\end{array}\right\}
$$

is an equivalence of categories, for the condition about the smallness of the $i$-head is automatically fulfilled, by the first part of the reasoning. Thus the existence and uniqueness result for $w$ is equivalent to that for $s_{i} w$. At this point, an induction concludes the proof.

Since the datum of $w \lambda$ allows us to recover $\lambda$, we may denote by $\widehat{N}(w \lambda)$ the $\Pi(\widehat{Q})$-module whose existence and uniqueness is asserted by Theorem 3.4. The following easy proposition studies the behaviour of these modules under the reflection functors.

Proposition 3.5. Let $\gamma$ be a weight and let $i \in I$. If $\left\langle\gamma, \alpha_{i}\right\rangle \leq 0$, then

$$
\Sigma_{i} \widehat{N}(\gamma) \cong \widehat{N}\left(s_{i} \gamma\right), \quad \Sigma_{i} \widehat{N}\left(s_{i} \gamma\right) \cong \widehat{N}\left(s_{i} \gamma\right) \quad \text { and } \quad \Sigma_{i}^{*} \widehat{N}\left(s_{i} \gamma\right) \cong \widehat{N}(\gamma)
$$

Proof. The first relation is a direct consequence of the proof of Theorem 3.4, to see it, it suffices to write $\gamma=w \lambda$ with $\lambda$ antidominant and $w \in W$ such that $\ell\left(s_{i} w\right)>\ell(w)$.

Lemma 2.6 then gives the second relation:

$$
\Sigma_{i} \widehat{N}\left(s_{i} \gamma\right) \cong \Sigma_{i}^{2} \widehat{N}(\gamma) \cong \Sigma_{i} \widehat{N}(\gamma) \cong \widehat{N}\left(s_{i} \gamma\right)
$$


Finally, the third relation comes from Proposition 2.5] and from the fact that $\widehat{N}(\gamma)$ has trivial $i$-head:

$$
\Sigma_{i}^{*} \widehat{N}\left(s_{i} \gamma\right) \cong \Sigma_{i}^{*} \Sigma_{i} \widehat{N}(\gamma) \cong \widehat{N}(\gamma)
$$

To conclude this section, we record the following consequence of Theorem 3.4 and Lemma 3.2. for any weight $\gamma$ and any $i \in I$,

$$
\left\{\begin{array}{l}
\operatorname{dim} \operatorname{Hom}_{\Pi(\widehat{Q})}\left(\widehat{N}(\gamma), S_{i}\right)=\max \left(0,\left\langle\gamma, \alpha_{i}\right\rangle\right), \\
\operatorname{dim} \operatorname{Ext}_{\Pi(\widehat{Q})}^{1}\left(\widehat{N}(\gamma), S_{i}\right)=\max \left(0,-\left\langle\gamma, \alpha_{i}\right\rangle\right) .
\end{array}\right.
$$

3.4. The modules $N(\gamma)$. Let $\lambda$ be an antidominant weight and let $w \in W$. Looking at the proofs of Lemma 3.3 (ii) and Theorem 3.4 one easily proves that if $\ell\left(s_{i} w\right)>\ell(w)$, then there is a canonical monomorphism $\widehat{N}(w \lambda) \hookrightarrow \widehat{N}\left(s_{i} w \lambda\right)$. (Alternatively, one can use Lemma 2.6 and Proposition 3.5.) In particular, $\widehat{N}(\lambda)$ is contained in each $\widehat{N}(w \lambda)$. Examining the dimension-vectors, one sees that $\widehat{N}(\lambda)$ is supported at the prime vertices and that the inclusion $\widehat{N}(\lambda) \hookrightarrow \widehat{N}(w \lambda)$ is an isomorphism at the prime vertices. Therefore the arrows $d_{i}^{*}$ act as the zero map on the module $\widehat{N}(w \lambda)$. We set $N(w \lambda)=\widehat{N}(w \lambda) / \widehat{N}(\lambda)$.

Now a $\Pi(Q)$-module $T$ can be viewed as a $\Pi(\widehat{Q})$-module by setting $T_{i^{\prime}}=0$ for all $i \in I$. Conversely, the $\Pi(\widehat{Q})$-module $N(w \lambda)$ can be viewed as a $\Pi(Q)$-module, because it is supported only on non-primed vertices. In this context, there are natural isomorphisms

$$
\operatorname{Hom}_{\Pi(\widehat{Q})}(\widehat{N}(w \lambda), T) \cong \operatorname{Hom}_{\Pi(\widehat{Q})}(N(w \lambda), T) \cong \operatorname{Hom}_{\Pi(Q)}(N(w \lambda), T) .
$$

We are now in a position to prove Theorem 3.1

Proof of Theorem 3.1. Assertion (i) is trivial.

Consider now the assertion (ii) and write $\gamma=-w \omega_{i}$, with $i \in I$ and $w \in W$. Set $M=\widehat{N}(\gamma)$; this is a stable $\Pi(\widehat{Q})$-module with dimension-vector $\left(\gamma+\omega_{i},-\omega_{i}\right)$. Then the vector space $\bigoplus_{j \in I} M_{j^{\prime}}$ coincides with the module $\widehat{N}\left(-\omega_{i}\right)$, so has dimension one and is concentrated at vertex $i^{\prime}$. On the other hand, the $\Pi(Q)$-module $N(\gamma)$ identifies with the vector space $\bigoplus_{i \in I} M_{i}$. In particular, the dimension-vector of $N(\gamma)$ is $\gamma+\omega_{i}$. For each $j \in I$, the $j$-socle of $M$ is the intersection of the $j$-socle of $N(\gamma)$ with $\operatorname{ker} M_{d_{j}}$. The stability of $M$ therefore implies that the socle of $N(\gamma)$ is concentrated at vertex $i$ and at most one-dimensional. Since $N(\gamma) \neq 0$, this socle cannot be zero, and we conclude that $\operatorname{soc} N(\gamma) \cong S_{i}$. The module $N(\gamma)$ thus enjoys the properties stated in assertion (ii). Conversely, let $N$ be a $\Pi(Q)$-module that enjoys these properties. One can then construct a stable $\Pi(\widehat{Q})$-module $M$ with dimension-vector $\left(\gamma+\omega_{i},-\omega_{i}\right)$ as follows: $M_{j}=N_{j}$ for all $j \in I, M_{h}=N_{h}$ for all $h \in H, M_{i^{\prime}}=\operatorname{soc}_{i} N, M_{d_{i}}: N_{i} \rightarrow \operatorname{soc}_{i} N$ is any linear map which extends the identity of $\operatorname{soc}_{i} N$, and the remaining spaces and arrows are zero. By the uniqueness in Theorem 3.4, $M$ is isomorphic to $\widehat{N}(\gamma)$, and we conclude that $N$ is isomorphic to $N(\gamma)$. Assertion (ii) is proved.

Finally, let $\lambda$ and $\mu$ be two antidominant weights and let $w \in W$. Then we have an isomorphism $\widehat{N}(w \lambda) \oplus \widehat{N}(w \mu) \cong \widehat{N}(w(\lambda+\mu))$, again by the uniqueness in Theorem 3.4. From there, it follows easily that $N(w \lambda) \oplus N(w \mu) \cong N(w(\lambda+\mu))$, which is the content of (iii). 
The modules $N(\gamma)$ have already been studied at great length by Geiß, Leclerc and Schröer, who have shown their relevance to the theory of cluster algebras. More precisely, the reflection functors $\Sigma_{i}$ can be seen as partial inverses to the functors $\mathcal{E}_{i}$ defined in section 5 of [9]. The key to this interpretation is Proposition 3.5] and the following result.

Proposition 3.6. The projective cover of $S_{i}$ in $\Pi(Q)$-mod is $N\left(\omega_{i}\right)$.

Proof. Consider a simple $\Pi(Q)$-module $S_{j}$, with $j \in I$. In the exact sequence

$$
\operatorname{Hom}_{\Pi(\widehat{Q})}\left(\widehat{N}\left(w_{0} \omega_{i}\right), S_{j}\right) \rightarrow \operatorname{Ext}_{\Pi(\widehat{Q})}^{1}\left(N\left(\omega_{i}\right), S_{j}\right) \rightarrow \operatorname{Ext}_{\Pi(\widehat{Q})}^{1}\left(\widehat{N}\left(\omega_{i}\right), S_{j}\right),
$$

the two extreme terms are zero, thanks to the equations (3.1). Hence the middle term is also zero. Observing that a non-split extension of $N\left(\omega_{i}\right)$ by $S_{j}$ in the category $\Pi(Q)$-mod would also be non-split in $\Pi(\widehat{Q})$-mod, we see that

$$
\operatorname{Ext}_{\Pi(Q)}^{1}\left(N\left(\omega_{i}\right), S_{j}\right)=0 .
$$

We conclude that $\operatorname{Ext}_{\Pi(Q)}^{1}\left(N\left(\omega_{i}\right), T\right)=0$ for any object $T$ in the category $\Pi(Q)$ mod, because such a $T$ has always a finite filtration with subquotients $S_{j}$.

Thus $N\left(\omega_{i}\right)$ is a projective $\Pi(Q)$-module, that is, is the projective cover of its head. On the other hand, the equality

$$
\operatorname{dim} \operatorname{Hom}_{\Pi(Q)}\left(N\left(\omega_{i}\right), S_{j}\right)=\operatorname{dim} \operatorname{Hom}_{\Pi(\widehat{Q})}\left(\widehat{N}\left(\omega_{i}\right), S_{j}\right)=\left\langle\alpha_{j}, \omega_{i}\right\rangle
$$

shows that the head of $N\left(\omega_{i}\right)$ is $S_{i}$.

We conclude this section by noting that in [14, Sadanand and the second author present a complete and explicit description of the modules $N(\gamma)$ in type A.

\section{Pseudo-Weyl polytopes}

For any weight $\gamma$ and any $\Pi(Q)$-module $T$, we set

$$
D_{\gamma}(T)=\operatorname{dim} \operatorname{Hom}_{\Pi(Q)}(N(\gamma), T) .
$$

Thus $D_{\gamma}(T)$ is always non-negative and $D_{\gamma}(T)=0$ if $\gamma$ is antidominant.

In this section, we study these functions $D_{\gamma}$. We first note the following consequence of Theorem 3.1.(iii)] $D_{\gamma+\delta}=D_{\gamma}+D_{\delta}$ whenever $\gamma$ and $\delta$ belong to the same Weyl chamber. This observation prompts us to pay a particular attention to the so-called chamber weights, that is, weights of the form $\gamma=w \omega_{i}$, with $w \in W$ and $i \in I$.

\subsection{Edge and other relations.}

Proposition 4.1. Let $\gamma$ be a weight, let $i \in I$ and let $T$ be a $\Pi(Q)$-module. If $\left\langle\gamma, \alpha_{i}\right\rangle \leq 0$, then

$$
\begin{aligned}
D_{\gamma}(T) & =D_{s_{i} \gamma}\left(\Sigma_{i} T\right), \\
D_{s_{i} \gamma}(T) & =D_{\gamma}\left(\Sigma_{i}^{*} T\right)-\left\langle\gamma, \underline{\operatorname{dim}} \operatorname{soc}_{i} T\right\rangle, \\
D_{\gamma}(T) & =D_{\gamma}\left(\Sigma_{i}^{*} \Sigma_{i} T\right) .
\end{aligned}
$$

Proof. We can regard $T$ as a module over either $\Pi(Q)$ or $\Pi(\widehat{Q})$; this does not affect the formation of $\Sigma_{i} T$. Having noticed this, equation (4.1) follows from the isomorphisms

$$
\operatorname{Hom}_{\Pi(\widehat{Q})}(\widehat{N}(\gamma), T) \cong \operatorname{Hom}_{\Pi(\widehat{Q})}\left(\Sigma_{i}^{*} \widehat{N}\left(s_{i} \gamma\right), T\right) \cong \operatorname{Hom}_{\Pi(\widehat{Q})}\left(\widehat{N}\left(s_{i} \gamma\right), \Sigma_{i} T\right)
$$


provided by Propositions 3.5 and 2.5 .

Replacing $T$ by $\Sigma_{i}^{*} T$ in this equality (4.1) yields

$$
D_{\gamma}\left(\Sigma_{i}^{*} T\right)=D_{s_{i} \gamma}\left(\Sigma_{i} \Sigma_{i}^{*} T\right)
$$

Now the equations (3.1) say that

$$
\operatorname{dim} \operatorname{Hom}_{\Pi(\widehat{Q})}\left(\widehat{N}\left(s_{i} \gamma\right), S_{i}\right)=-\left\langle\gamma, \alpha_{i}\right\rangle \quad \text { and } \quad \operatorname{Ext}_{\Pi(\widehat{Q})}^{1}\left(\widehat{N}\left(s_{i} \gamma\right), S_{i}\right)=0 .
$$

Equation (4.2) then follows by applying the functor $\operatorname{Hom}_{\Pi(\widehat{Q})}\left(\widehat{N}\left(s_{i} \gamma\right), ?\right)$ to the short exact sequence

$$
0 \rightarrow \operatorname{soc}_{i} T \rightarrow T \rightarrow \Sigma_{i} \Sigma_{i}^{*} T \rightarrow 0 .
$$

Finally, (4.3) is obtained by writing (4.2) for the $\Pi(Q)$-module $\Sigma_{i} T$, which has trivial $i$-socle.

Let $a_{i j}=\left\langle\alpha_{i}, \alpha_{j}\right\rangle$ be the entries of the Cartan matrix of the Dynkin diagram $\Gamma$.

Proposition 4.2 (Edge relations). Let $T$ be a $\Pi(Q)$-module. Then for each $i \in I$ and each $w \in W$, one has

$$
D_{-w \omega_{i}}(T)+D_{-w s_{i} \omega_{i}}(T)+\sum_{\substack{j \in I \\ j \neq i}} a_{i j} D_{-w \omega_{j}}(T) \geq 0 .
$$

Proof. We fix $i \in I$. The equality $s_{i} \omega_{i}=\omega_{i}-\alpha_{i}$ can be rewritten as

$$
\omega_{i}+s_{i} \omega_{i}+\sum_{\substack{j \in I \\ j \neq i}} a_{i j} \omega_{j}=0
$$

Let us call $L_{w}(T)$ the left-hand side of our desired edge relation. We want to show that $L_{w}(T) \geq 0$ for all $w \in W$. Since $L_{w}(T)=L_{w s_{i}}(T)$, we may restrict our attention to the elements $w$ such that $\ell\left(w s_{i}\right)>\ell(w)$.

Take such a $w$, assume that it has positive length, and write $w=s_{k} v$ with $k \in I$ and $\ell(v)=\ell(w)-1$. Observing that $\ell\left(s_{k} v\right)>\ell(v)$ and $\ell\left(s_{k} v s_{i}\right)>\ell(w)=$ $\ell(v)+1 \geq \ell\left(v s_{i}\right)$, a straightforward computation based on equations (4.2) and (4.4) shows then that

$$
L_{w}(T)=L_{v}\left(\Sigma_{k}^{*} T\right) .
$$

By induction, we thus see that if $s_{k_{1}} \cdots s_{k_{r}}$ is a reduced decomposition of $w$, then

$$
L_{w}(T)=L_{1}\left(\Sigma_{k_{r}}^{*} \cdots \Sigma_{k_{1}}^{*} T\right) .
$$

Since $D_{\gamma}(M)=0$ for all antidominant weight $\gamma$ and all $\Pi(Q)$-modules $M$, we finally obtain

$$
L_{w}(T)=D_{-s_{i} \omega_{i}}\left(\Sigma_{k_{r}}^{*} \cdots \Sigma_{k_{1}}^{*} T\right) \geq 0 .
$$

Proposition 4.3. Let $\gamma$ be a weight and $T$ be $a \Pi(Q)$-module. Then

$$
D_{\gamma}(T)-D_{-\gamma}\left(T^{*}\right)=\langle\gamma, \underline{\operatorname{dim}} T\rangle .
$$

Proof. Let $i \in I$. By Proposition 3.6, $D_{\omega_{i}}(T)$ is equal to the Jordan-Hölder multiplicity of $S_{i}$ in $T$, and therefore to $\left\langle\omega_{i}, \underline{\operatorname{dim}} T\right\rangle$. This fact implies the desired equality in the case $\gamma=-\omega_{i}$. The case where $\gamma$ is antidominant then follows from Theorem 3.1 (iii)

Fix an antidominant weight $\lambda$. For $w \in W$, call $\mathscr{E}_{w}(T)$ the desired equality for $\gamma=w \lambda$. Thus the first part of the proof establishes $\mathscr{E}_{1}(T)$ for any $T$. 
Take $w \in W$ and $i \in I$ such that $\ell\left(s_{i} w\right)>\ell(w)$. The third equality in Proposition 3.5 gives

$$
\Sigma_{i}^{*} \widehat{N}(-w \lambda) \cong \widehat{N}\left(-s_{i} w \lambda\right)
$$

By Proposition 2.5 and Remark 2.4 (i), it follows that

$$
\begin{aligned}
\operatorname{Hom}_{\Pi(\widehat{Q})}\left(\widehat{N}\left(-s_{i} w \lambda\right), T^{*}\right) & \cong \operatorname{Hom}_{\Pi(\widehat{Q})}\left(\widehat{N}(-w \lambda), \Sigma_{i} T^{*}\right) \\
& \cong \operatorname{Hom}_{\Pi(\widehat{Q})}\left(\widehat{N}(-w \lambda),\left(\Sigma_{i}^{*} T\right)^{*}\right),
\end{aligned}
$$

whence

$$
D_{-s_{i} w \lambda}\left(T^{*}\right)=D_{-w \lambda}\left(\left(\Sigma_{i}^{*} T\right)^{*}\right) .
$$

A straightforward computation based on equation (4.2) and on

$$
\underline{\operatorname{dim}} T=\underline{\operatorname{dim}} \Sigma_{i} \Sigma_{i}^{*} T+\underline{\operatorname{dim}} \operatorname{soc}_{i} T=s_{i}\left(\underline{\operatorname{dim}} \Sigma_{i}^{*} T\right)+\underline{\operatorname{dim}} \operatorname{soc}_{i} T
$$

shows then that $\mathscr{E}_{s_{i} w}(T)$ is equivalent to $\mathscr{E}_{w}\left(\Sigma_{i}^{*} T\right)$.

We conclude the proof by an immediate induction on $\ell(w)$.

Remark 4.4. (i) It is worthwhile to record the starting point for the induction in the proof of Proposition 4.3. if $\gamma$ is dominant, then

$$
\operatorname{dim} \operatorname{Hom}_{\Pi(Q)}(N(\gamma), T)=D_{\gamma}(T)=\langle\gamma, \underline{\operatorname{dim}} T\rangle .
$$

(ii) Let $\gamma$ be a non-antidominant chamber weight. Recall that $N(\gamma)$ is characterized by having dimension-vector $\gamma+\omega_{i}$ and socle $S_{i}$. Hence $N(\gamma)^{*}$ is characterized by having dimension vector $\gamma+\omega_{i}$ and head $S_{i}$. Note also that

$$
\operatorname{Hom}(N(\gamma), T)=\operatorname{Hom}\left(T^{*}, N(\gamma)^{*}\right) .
$$

Thus, we could have defined $D_{\gamma}$ in terms of morphisms into modules with one-dimensional head, rather than out of modules with one-dimensional socle.

(iii) Let $\gamma$ be a non-antidominant chamber weight. We can write $\gamma$ as the difference $\gamma_{0}-\gamma_{1}$ of two dominant weights with disjoint "supports": a fundamental weight $\omega_{i}$ may appear in $\gamma_{0}$ or in $\gamma_{1}$, but not in both. Let us set $P_{0}=N\left(\gamma_{0}\right)$ and $P_{1}=N\left(\gamma_{1}\right)$. Proposition 3.6 and Theorem 3.1 (iii) imply that $P_{0}$ and $P_{1}$ are projective modules. Using Theorem 3.1 (ii) equation (3.1) and Crawley-Boevey's formula, one can show that the minimal projective resolution of $N(\gamma)$ starts with

$$
\cdots \rightarrow P_{1} \rightarrow P_{0} \rightarrow N(\gamma) \rightarrow 0 .
$$

For any $\Pi(Q)$-module $T$, Corollary IV.4.3 in [2, Remark 4.4 (i)] above and Proposition 4.3 then give

$$
\begin{aligned}
\operatorname{dim} \operatorname{Hom}_{\Pi(Q)}(N(\gamma), T)-\operatorname{dim} & \operatorname{Hom}_{\Pi(Q)}(T, D \operatorname{Tr} N(\gamma)) \\
& =\operatorname{dim} \operatorname{Hom}_{\Pi(Q)}\left(P_{0}, T\right)-\operatorname{dim} \operatorname{Hom}_{\Pi(Q)}\left(P_{1}, T\right) \\
& =\left\langle\gamma_{0}, \underline{\operatorname{dim}} T\right\rangle-\left\langle\gamma_{1}, \underline{\operatorname{dim}} T\right\rangle \\
& =\langle\gamma, \underline{\operatorname{dim}} T\rangle \\
& =D_{\gamma}(T)-D_{-\gamma}\left(T^{*}\right),
\end{aligned}
$$

where $D$ Tr denotes the Auslander-Reiten translation. Remark 4.4 (ii) says that $D_{-\gamma}\left(T^{*}\right)=\operatorname{Hom}\left(T, N(-\gamma)^{*}\right)$, so

$$
\operatorname{dim} \operatorname{Hom}_{\Pi(Q)}(T, D \operatorname{Tr} N(\gamma))=\operatorname{dim} \operatorname{Hom}_{\Pi(Q)}\left(T, N(-\gamma)^{*}\right)
$$


holds for any $T$. We conjecture that in fact $N(-\gamma)^{*} \cong D \operatorname{Tr} N(\gamma)$.

4.2. Pseudo-Weyl polytopes. Following Appendix A in [13, we recall the notion of pseudo-Weyl polytope.

Let $V$ be a $\mathbb{R}$-vector space and let $V^{*}$ be its dual. To a non-empty compact convex subset $P$ of $V$, we associate its support function $\psi_{P}: V^{*} \rightarrow \mathbb{R}$ : it maps a linear form $\alpha \in V^{*}$ to the maximal value $\alpha$ takes on $P$. Then $\psi_{P}$ is a sublinear function on $V^{*}$. One can recover $P$ from the datum of $\psi_{P}$ by the Hahn-Banach theorem

$$
P=\left\{v \in V \mid \forall \alpha \in V^{*},\langle v, \alpha\rangle \leq \psi_{P}(\alpha)\right\},
$$

and the map $P \mapsto \psi_{P}$ is a bijection from the set of all non-empty compact convex subsets of $V$ onto the set of all sublinear functions on $V^{*}$ (see for instance Chapter $\mathrm{C}$ in [11). If $P$ is a polytope, then its support function is piecewise linear. More precisely, the maximal regions of linearity of $\psi_{P}$ are exactly the maximal cones of the dual fan of $P$ : for each vertex $v$ of $P$, the support function $\psi_{P}$ is linear on $\left\{\alpha \in V^{*} \mid \psi_{P}(\alpha)=\langle v, \alpha\rangle\right\}$.

We now specialize to the case $V=Q \otimes_{\mathbb{Z}} \mathbb{R}$, the real vector space spanned by the root system of $\mathfrak{g}$. We say that a polytope $P \subseteq V$ is a pseudo-Weyl polytope if its support function is linear on each Weyl chamber. Then to each Weyl chamber $C$ corresponds a vertex $v_{C}$ of $P$, defined by the condition

$$
\forall \gamma \in C, \quad \psi_{P}(\gamma)=\left\langle\gamma, v_{C}\right\rangle
$$

One gets all the vertices of $P$ in this way (possibly with repetitions, since the Weyl fan can be finer than the dual fan of $P$ ). For $w \in W$, we denote by $\mu_{w}$ the vertex that corresponds to the chamber formed by the elements $w \lambda$ with $\lambda$ antidominant. This indexed collection $\left(\mu_{w}\right)_{w \in W}$ is called the vertex datum of $P$.

Let $\Gamma=\left\{w \omega_{i} \mid i \in I, w \in W\right\}$ denote the set of chamber weights. Then each Weyl chamber is spanned by a subset of $\Gamma$, which implies that the support function of a pseudo-Weyl polytope is entirely characterized by its values on $\Gamma$. A pseudo-Weyl polytope $P$ is thus characterized by the collection of real numbers $\left(\psi_{P}(\gamma)\right)_{\gamma \in \Gamma} ;$ concretely, these numbers describe the position of the facets of $P$ :

$$
P=\left\{v \in V \mid \forall \gamma \in \Gamma,\langle\gamma, v\rangle \leq \psi_{P}(\gamma)\right\} .
$$

This collection of real numbers is called the hyperplane datum of $P$.

Conversely, we can start from a collection of numbers $\left(A_{\gamma}\right)_{\gamma \in \Gamma}$ and ask ourselves whether there exists a sublinear function $\psi: V^{*} \rightarrow \mathbb{R}$, linear on each Weyl chamber, such that $\psi(\gamma)=A_{\gamma}$ for each $\gamma \in \Gamma$; in other words, whether $\left(A_{\gamma}\right)_{\gamma \in \Gamma}$ is the hyperplane datum of a pseudo-Weyl polytope. The answer is given in Lemma A.5 in [13]: a necessary and sufficient condition is that $\left(A_{\gamma}\right)_{\gamma \in \Gamma}$ satisfy the edge inequalities

$$
A_{-w \omega_{i}}+A_{-w s_{i} \omega_{i}}+\sum_{\substack{j \in I \\ j \neq i}} a_{i j} A_{-w \omega_{j}} \geq 0
$$

for all $i \in I$ and $w \in W$. When this condition is fulfilled, the vertex datum $\left(\mu_{w}\right)_{w \in W}$ of the corresponding pseudo-Weyl polytope is characterized by the set of equations

$$
\left\langle-w \omega_{i}, \mu_{w}\right\rangle=A_{-w \omega_{i}},
$$

and the left-hand side of (4.5) is equal to the length $c$ of the edge between the vertices $\mu_{w}$ and $\mu_{w s_{i}}$, defined by the equation $\mu_{w s_{i}}-\mu_{w}=c w \alpha_{i}$, by formula (8) in 
[13. From (4.6), we see that all the weights $\mu_{w}$ belong to $Q$ if and only if all the numbers $A_{\gamma}$ are integers.

Note that in this paper, we use a different labelling of the vertices by Weyl group elements, as compared to [13. To translate between the two notions, just set $\nu_{w}=\mu_{w w_{0}}$, where $\left(\nu_{w}\right)_{w \in W}$ denotes a vertex datum from [13.

4.3. GGMS strata. We keep the conventions of the previous section. For each $\Pi(Q)$-module $T$, we have the collection of integers $\left(D_{\gamma}(T)\right)_{\gamma \in \Gamma}$. Proposition 4.2 shows that this collection satisfies the edge inequalities. It is thus the hyperplane datum of a pseudo-Weyl polytope, namely

$$
\operatorname{Pol}(T)=\left\{v \in V \mid \forall \gamma \in \Gamma,\langle\gamma, v\rangle \leq D_{\gamma}(T)\right\} .
$$

Thus $D_{\gamma}(T)$ is the value $\psi_{\operatorname{Pol}(T)}(\gamma)$ of the support function, for each $\gamma \in \Gamma$, and indeed for each $\gamma \in P$, thanks to Theorem 3.1 (iii). The vertex datum $\left(\mu_{w}(T)\right)_{w \in W}$ of $\operatorname{Pol}(T)$ can be obtained from the numbers $D_{\gamma}(T)$ by the formula (4.6), with $D_{\gamma}(T)$ instead of $A_{\gamma}$. Using Remark 4.4 (i), we note that $\mu_{1}(T)=0$ and $\mu_{w_{0}}(T)=$ $\underline{\operatorname{dim}} T$.

Remark 4.5. Proposition 4.3 implies that for any submodule $U \subseteq T$, the inequality

$$
\langle\gamma, \underline{\operatorname{dim}} U\rangle=D_{\gamma}(U)-D_{-\gamma}\left(U^{*}\right) \leq D_{\gamma}(U) \leq D_{\gamma}(T)
$$

holds for each $\gamma \in \Gamma$; it follows that $\underline{\operatorname{dim}} U \in \operatorname{Pol}(T)$. In [3], we will show that, in fact, $\operatorname{Pol}(T)$ is the convex hull of the dimension-vectors of all submodules of $T$; moreover, we will describe precisely the submodules $U$ such that $\operatorname{dim} U$ is an extremal point of $\operatorname{Pol}(T)$.

Conversely, for each dimension-vector $\nu$ and each pseudo-Weyl polytope $P$ whose vertex datum satisfies $\mu_{1}=0$ and $\mu_{w_{0}}=\nu$, we may consider the set of all points in $\Lambda(\nu)$ whose polytope is $P$. We obtain in this way a stratification of $\Lambda(\nu)$ whose strata are labelled by pseudo-Weyl polytopes of weight $\nu$. In analogy with the situation for the affine Grassmannian (see section 2.4 of [13]), we call these the GGMS strata of $\Lambda(\nu)$. Later in this paper, we will see that the strata of biggest dimension (whose closures are the components) are those labelled by MV polytopes - exactly as in the case of the affine Grassmannian. On the other hand, we do not know how to analyse the other GGMS strata and compare them with the corresponding GGMS strata in the affine Grassmannian.

To conclude, let us emphasize the meaning of Proposition 4.3 for any $\Pi(Q)$ module $T$, the pseudo-Weyl polytope $\operatorname{Pol}\left(T^{*}\right)$ is the image of $\operatorname{Pol}(T)$ under the involution $x \mapsto \underline{\operatorname{dim}} T-x$.

\section{Crystal structure}

The combinatorics of representations of complex semi-simple Lie algebras is in part controlled by Kashiwara crystals; see [15] for a survey of this theory. The crystals $B(\lambda)$ of the finite-dimensional representations of $\mathfrak{g}$ are "contained" in a big crystal $B(-\infty)$, which is the crystal of the positive part $U(\mathfrak{n})$ of $U(\mathfrak{g})$. In [16], Kashiwara and Saito gave a characterization of $B(-\infty)$ as the unique highest weight crystal having an involution with certain specific properties.

Let us denote by $\operatorname{Irr} X$ the set of irreducible components of an algebraic variety $X$. The set $\mathbf{B}=\bigsqcup_{\nu \in Q_{+}} \operatorname{Irr} \Lambda(\nu)$ can be endowed with the structure of a crystal with an involution, which turns out to be isomorphic to $B(-\infty)$. Thus to an element 
$b \in B(-\infty)$, of weight $\nu$, corresponds $\Lambda_{b} \in \operatorname{Irr} \Lambda(\nu)$. Our aim in this section is to show that the reflection functor $\Sigma_{i}$ has a crystal counterpart, namely the map denoted by $S_{i}$ in [16]. (We will adopt this notation $S_{i}$, though it brings some confusion with our previous notation for the simple quiver representations.)

5.1. The crystal structure. We begin by recalling the crystal structure on $\mathbf{B}$, first defined by Lusztig in 19, section 8.

Let $\nu \in Q_{+}$. As in section 2.1, we set $M_{i}=K^{\nu_{i}}$ for each $i \in I$. Any point $\left(M_{a}\right) \in \Lambda(\nu)$ then defines a $\Pi(Q)$-module with dimension-vector $\nu$. An element $Z \in \operatorname{Irr} \Lambda(\nu)$ is said to have weight $\operatorname{wt}(Z)=\nu$.

The usual isomorphism between the vector spaces $M_{i}$ and their duals allows to view the involution $*$ on $\Pi(Q)$-modules as an operation on $\Lambda(\nu)$ (it just amounts to the transposition and subsequent relabelling of the matrices $\left.M_{a}\right)$. This operation induces an involution on $\operatorname{Irr} \Lambda(\nu)$. We thus obtain a weight preserving involution on $\mathbf{B}$, which we denote again by $*$.

For $i \in I$ and $c \in \mathbb{N}$, let

$$
\Lambda(\nu)_{i, c}=\left\{\left(M_{a}\right) \mid \operatorname{dimhd}_{i} M=c\right\} .
$$

These sets form a partition of $\Lambda(\nu)$ into locally closed subsets. For any irreducible component $Z$ of $\Lambda(\nu)$, there is thus one value of $c$ such that $Z \cap \Lambda(\nu)_{i, c}$ is open and dense in $Z$. We then write $\varphi_{i}(Z)=c$ and $\varepsilon_{i}(Z)=c-\left\langle\alpha_{i}, \nu\right\rangle$.

In this context, we note that $\Lambda(\nu)$ and each subset $\Lambda(\nu)_{i, c}$ have pure dimension equal to $\operatorname{dim} G(\nu)-\langle\nu, \nu\rangle / 2$; see Theorem 8.7 in [19]. Therefore the map $Z \mapsto$ $Z \cap \Lambda(\nu)_{i, c}$ gives a bijection

$$
\left\{Z \in \operatorname{Irr} \Lambda(\nu) \mid \varphi_{i}(Z)=c\right\} \rightarrow \operatorname{Irr} \Lambda(\nu)_{i, c} .
$$

Again, let $i \in I$. Given $c \in \mathbb{N}$, let us denote by $\Omega(\nu, i, c)$ the set of triples $\left(\left(M_{a}\right),\left(N_{a}\right), g\right)$ such that $\left(M_{a}\right) \in \Lambda(\nu)_{i, 0},\left(N_{a}\right) \in \Lambda\left(\nu+c \alpha_{i}\right)_{i, c}$ and $g: M \rightarrow N$ is an injective morphism of $\Pi(Q)$-modules. We can then form the diagram

$$
\Lambda(\nu)_{i, 0} \stackrel{p}{\leftarrow} \Omega(\nu, i, c) \stackrel{q}{\rightarrow} \Lambda\left(\nu+c \alpha_{i}\right)_{i, c},
$$

where $p$ and $q$ are the first and second projection. Then $p$ is a locally trivial fibration, with a smooth and connected fiber, and $q$ is a principal $G(\nu)$-bundle. Thus $p$ and $q$ define bijections

$$
\operatorname{Irr} \Lambda(\nu)_{i, 0} \longleftrightarrow \operatorname{Irr} \Omega(\nu, i, c) \longleftrightarrow \operatorname{Irr} \Lambda\left(\nu+c \alpha_{i}\right)_{i, c} .
$$

We therefore obtain mutually inverse bijections

$$
\left\{Z \in \operatorname{Irr} \Lambda(\nu) \mid \varphi_{i}(Z)=0\right\} \underset{\tilde{f}_{i}^{\max }}{\stackrel{\tilde{e}_{i}^{c}}{\rightleftarrows}}\left\{Z \in \operatorname{Irr} \Lambda\left(\nu+c \alpha_{i}\right) \mid \varphi_{i}(Z)=c\right\},
$$

whose data is equivalent to the data of the usual structure maps for a crystal

$$
\left\{Z \in \operatorname{Irr} \Lambda\left(\nu+c \alpha_{i}\right) \mid \varphi_{i}(Z)=c\right\} \underset{\tilde{f}_{i}}{\stackrel{\tilde{e}_{i}}{\rightleftarrows}}\left\{Z \in \operatorname{Irr} \Lambda\left(\nu+(c+1) \alpha_{i}\right) \mid \varphi_{i}(Z)=c+1\right\} .
$$

It is known that the maps wt, $\varepsilon_{i}, \varphi_{i}, \tilde{e}_{i}$ and $\tilde{f}_{i}$ endow $\mathbf{B}$ with the structure of a crystal. Using the involution $*$, Kashiwara and Saito proved the existence of an isomorphism $b \mapsto \Lambda_{b}$ from $B(-\infty)$ onto $\mathbf{B}$ (Theorem 5.3.2 in [16]). This isomorphism is unique since $B(-\infty)$ has no automorphism. As usual, one writes $\tilde{e}_{i}^{*}=* \tilde{e}_{i} *$ and $\tilde{f}_{i}^{*}=* \tilde{f}_{i} *$. 
For $\nu \in Q_{+}, i \in I$ and $c \in \mathbb{N}$, let us set $\Lambda(\nu)_{i, c}^{\times}=\Lambda(\nu)_{i, c} \cap\left(\Lambda(\nu)_{i, 0}\right)^{*}$. Assuming, moreover, that $0 \leq c \leq-\left\langle\alpha_{i}, \nu\right\rangle$, let us denote by $\Omega(\nu, i, c)^{\times}$the set of triples $\left(\left(M_{a}\right),\left(N_{a}\right), g\right)$ such that $\left(M_{a}\right) \in \Lambda(\nu)_{i, 0}^{\times},\left(N_{a}\right) \in \Lambda\left(\nu+c \alpha_{i}\right)_{i, c}^{\times}$and $g: M \rightarrow N$ is an injective morphism of $\Pi(Q)$-modules. By restriction, the diagram (5.1) yields

$$
\Lambda(\nu)_{i, 0}^{\times} \stackrel{p^{\times}}{\stackrel{(}{\longleftarrow}} \Omega(\nu, i, c)^{\times} \stackrel{q^{\times}}{\longrightarrow} \Lambda\left(\nu+c \alpha_{i}\right)_{i, c}^{\times} .
$$

Lemma 5.1. In the context above, $p^{\times}$is a locally trivial fibration, with a smooth and connected fiber, and $q^{\times}$is a principal $G(\nu)$-bundle.

Proof. Let us set $M_{i}=K^{\nu_{i}}, N_{i}=K^{\nu_{i}+c}$, and $M_{j}=N_{j}=K^{\nu_{j}}$ for $j \neq i$.

Let us first study the fibers of $p^{\times}$. We thus fix a point $\left(M_{a}\right) \in \Lambda(\nu)_{i, 0}^{\times}$and look at the set of pairs $\left(\left(N_{a}\right), g\right)$, where $\left(N_{a}\right) \in \Lambda\left(\nu+c \alpha_{i}\right)_{i, c}^{\times}$and $g: M \rightarrow N$ is an injective morphism. The isomorphisms of vector spaces $g_{j}: M_{j} \rightarrow N_{j}$ with $j \neq i$ can be freely chosen, and their datum determines the maps $N_{a}$ for all $a \in H$ such that $i \notin\{s(a), t(a)\}$. It thus remains to study the local situation around $i$.

We adopt the notations set up in section 2.2. The situation can be summarized by a commutative diagram

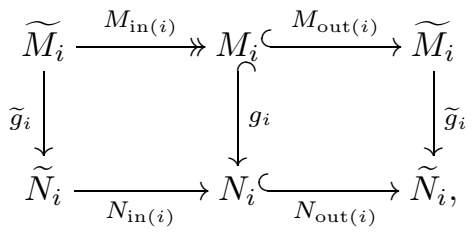

where $\widetilde{g}_{i}$ is an isomorphism of vector spaces. The injectivity of $M_{\text {out }(i)}$ and $N_{\text {out }(i)}$ follows from the fact that $\left(M_{a}\right) \in\left(\Lambda(\nu)_{i, 0}\right)^{*}$ and $\left(N_{a}\right) \in\left(\Lambda\left(\nu+c \alpha_{i}\right)_{i, 0}\right)^{*}$.

The image of the map $u=M_{\mathrm{out}(i)} M_{\mathrm{in}(i)}$ has the same dimension as $M_{i}$, namely $\nu_{i}$. The image of $\left(\widetilde{g}_{i}\right)^{-1} N_{\text {out }(i)}$ is a subspace $V$ of $\widetilde{M}_{i}$ with dimension $\nu_{i}+c$ and such that $\operatorname{im} u \subseteq V \subseteq \operatorname{ker} u$. In other words, $V / \operatorname{im} u$ can be chosen freely in the Grassmannian of $c$-dimensional subspaces of $\operatorname{ker} u / \operatorname{im} u$ (note that since $\operatorname{dim} \operatorname{im} u=$ $\operatorname{dim} M_{i}$ and $\operatorname{dim} \operatorname{ker} u=\operatorname{dim} \widetilde{M}_{i}-\operatorname{dim} M_{i}$, we have $\left.\operatorname{dim} \operatorname{ker} u / \operatorname{im} u=-\left\langle\alpha_{i}, \nu\right\rangle \geq c\right)$. The choice of such a $V$ and of an isomorphism of vector spaces $\bar{N}_{\text {out }(i)}: N_{i} \rightarrow \widetilde{g}_{i}(V)$ determines the remaining data $g_{i}, N_{\text {in(i) }}$ and $N_{\text {out }(i)}$. We conclude that the fiber of $p$ above $\left(M_{a}\right)$ has the structure of a principal $G\left(\nu+c \alpha_{i}\right)$-bundle over a Grassmannian. This Grassmannian depends smoothly on $\left(M_{a}\right)$, so we can conclude that $p^{\times}$is a locally trivial fibration, with a smooth and connected fiber.

The proof of the assertion about $q^{\times}$is similar, but simpler.

We can now write a commutative diagram

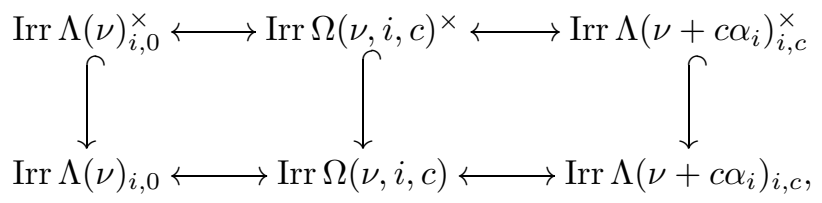

where the horizontal arrows are the bijections defined by the maps in the diagrams (5.2) and (5.1) and the vertical arrows are given by the open inclusions

$$
\Lambda(\nu)_{i, 0}^{\times} \subseteq \Lambda(\nu)_{i, 0}, \quad \Omega(\nu, i, c)^{\times} \subseteq \Omega(\nu, i, c) \quad \text { and } \quad \Lambda\left(\nu+c \alpha_{i}\right)_{i, c}^{\times} \subseteq \Lambda\left(\nu+c \alpha_{i}\right)_{i, c} .
$$


In particular, we see that if $0 \leq c \leq-\left\langle\alpha_{i}, \nu\right\rangle$, then $\tilde{e}_{i}^{c}$ and $\tilde{f}_{i}^{\max }$ restrict to bijections

$$
\left\{\begin{array}{l|l}
Z \in \operatorname{Irr} \Lambda(\nu) & \begin{array}{c}
\varphi_{i}(Z)=0 \\
\varphi_{i}\left(Z^{*}\right)=0
\end{array}
\end{array}\right\} \underset{\tilde{e}_{i}^{c}}{\rightleftarrows}\left\{\begin{array}{l|l}
Z \in \operatorname{Irr} \Lambda\left(\nu+c \alpha_{i}\right) & \begin{array}{c}
\varphi_{i}(Z)=c \\
\varphi_{i}\left(Z^{*}\right)=0
\end{array}
\end{array}\right\} .
$$

Remark 5.2. Taking into account the crystal isomorphism $\mathbf{B} \cong B(-\infty)$, we conclude that for any $b \in B(-\infty)$ and $c \in \mathbb{N}$,

$$
\left(\varphi_{i}(b)=\varphi_{i}\left(b^{*}\right)=0 \text { and } 0 \leq c \leq-\left\langle\alpha_{i}, \operatorname{wt}(b)\right\rangle\right) \Longrightarrow \varphi_{i}\left(\left(\tilde{e}_{i}^{c} b\right)^{*}\right)=0 .
$$

This result is indeed contained in Proposition 5.3.1 (1) in [16], which says that

$$
\varphi_{i}(b)=\max \left(\varphi_{i}\left(\left(\tilde{f}_{i}^{*}\right)^{\max } b\right),\left\langle\operatorname{wt}(b), \alpha_{i}\right\rangle-\varphi_{i}\left(b^{*}\right)\right)
$$

for each $b \in B(-\infty)$. A particular case of the latter formula is that for each $b \in B(-\infty)$ that satisfies $\varphi_{i}(b)=0$, the number $\varepsilon_{i}\left(b^{*}\right)=\varphi_{i}\left(b^{*}\right)-\left\langle\alpha_{i}\right.$, wt $\left.(b)\right\rangle$ is non-negative.

5.2. Reflection functors and crystal operations. Let us fix $i \in I$ for this whole section. In Corollary 3.4.8 in [29] (see also section 8.2 in [16]), Saito defines a bijection

$$
S_{i}:\left\{b \in B(-\infty) \mid \varphi_{i}(b)=0\right\} \rightarrow\left\{b \in B(-\infty) \mid \varphi_{i}\left(b^{*}\right)=0\right\}
$$

by the rule $S_{i}(b)=\tilde{e}_{i}^{\varepsilon_{i}\left(b^{*}\right)}\left(\tilde{f}_{i}^{*}\right)^{\max } b$. This operation plays the role of the simple reflection $s_{i}$, but at the crystal level. One can indeed check that

$$
\mathrm{wt}\left(S_{i} b\right)=\operatorname{wt}(b)+\left(\varepsilon_{i}\left(b^{*}\right)-\varphi_{i}\left(b^{*}\right)\right) \alpha_{i}=\mathrm{wt}(b)-\left\langle\alpha_{i}, \operatorname{wt}\left(b^{*}\right)\right\rangle \alpha_{i}=s_{i}(\operatorname{wt}(b)) .
$$

A deeper property is that $S_{i}$ implements at the crystal level the action of Lusztig's braid group automorphism $T_{i}=T_{i,-1}^{\prime}$ on the canonical basis; see [21] and Proposition 3.4.7 in [29]. We now relate this operation $S_{i}$ to the reflection functor $\Sigma_{i}$.

To do that, let us fix $\nu \in Q_{+}$and let us denote by $\Theta(\nu, i)$ the set of all triples $\left(\left(M_{a}\right),\left(N_{a}\right), h\right)$ such that $\left(M_{a}\right) \in \Lambda(\nu)_{i, 0},\left(N_{a}\right) \in\left(\Lambda\left(s_{i} \nu\right)_{i, 0}\right)^{*}$ and $h: N \rightarrow \Sigma_{i} M$ is an isomorphism. We can then form the diagram

$$
\Lambda(\nu)_{i, 0} \stackrel{r}{\leftarrow} \Theta(\nu, i) \stackrel{s}{\rightarrow}\left(\Lambda\left(s_{i} \nu\right)_{i, 0}\right)^{*},
$$

where $r$ and $s$ are the first and second projection.

There is an obvious action of $G\left(s_{i} \nu\right)$ on $\Theta(\nu, i)$, whose orbits are the fibers of $r$. In addition, the linear map $M_{\text {in }(i)}$ has always full rank when $\left(M_{a}\right) \in \Lambda(\nu)_{i, 0}$, so $\left(M_{a}\right) \mapsto \operatorname{ker} M_{\mathrm{in}(i)}$ is a continuous map from $\Lambda(\nu)_{i, 0}$ to the relevant Grassmannian of the vector space $\widetilde{M}_{i}$. With the help of local charts of the Grassmannian, we can then construct local homeomorphisms $t_{\alpha}: r^{-1}\left(U_{\alpha}\right) \cong U_{\alpha} \times G\left(s_{i} \nu\right)$, where $\left(U_{\alpha}\right)$ is an open covering of $\Lambda(\nu)_{i, 0}$, and prove that $r$ is a principal $G\left(s_{i} \nu\right)$ bundle.

A similar argument proves that $s$ is a principal $G(\nu)$-bundle; in fact, the remark after Proposition 2.5 shows that $M$ and $N$ play symmetric roles. Thus $r$ and $s$ are locally trivial fibrations with a smooth and connected fiber; therefore they define bijections

$$
\operatorname{Irr} \Lambda(\nu)_{i, 0} \longleftrightarrow \operatorname{Irr} \Theta(\nu, i) \longleftrightarrow \operatorname{Irr}\left(\left(\Lambda\left(s_{i} \nu\right)_{i, 0}\right)^{*}\right)
$$

Theorem 5.3. Let $b \in B(-\infty)$ of weight $\nu$ and such that $\varphi_{i}(b)=0$. In the notation above,

$$
r^{-1}\left(\Lambda_{b} \cap \Lambda(\nu)_{i, 0}\right)=s^{-1}\left(\Lambda_{S_{i} b} \cap\left(\Lambda\left(s_{i} \nu\right)_{i, 0}\right)^{*}\right) .
$$


Proof. A direct calculation shows that for any $\Pi(Q)$-modules $M$ and $N$ such that $\operatorname{hd}_{i} M=0, \operatorname{soc}_{i} N=0$ and $N \cong \Sigma_{i} M$, one has

$$
\operatorname{dim} M_{i}-\operatorname{dim} \operatorname{soc}_{i} M=\operatorname{dim} N_{i}-\operatorname{dim} \operatorname{hd}_{i} N .
$$

Now let $\mu \in Q_{+}$and let $c, d \in \mathbb{N}$ be such that $c+d=-\left\langle\alpha_{i}, \mu\right\rangle$. Set $\nu=\mu+c \alpha_{i}$; thus $s_{i} \nu=\mu+d \alpha_{i}$. Two $\Pi(Q)$-modules $M$ and $N$ such that

$$
\underline{\operatorname{dim}} M=\nu, \quad \operatorname{hd}_{i} M=0, \quad \underline{\operatorname{dim}} N=s_{i} \nu, \quad \operatorname{soc}_{i} N=0, \quad \Sigma_{i} M \cong N
$$

are then related by

$$
\operatorname{dim} \operatorname{soc}_{i} M=c \Longleftrightarrow \operatorname{dimhd}_{i} N=d .
$$

The diagram (5.3) thus restricts to

$$
\Lambda(\nu)_{i, 0} \cap\left(\Lambda(\nu)_{i, c}\right)^{*} \leftarrow \Theta(\nu, i)_{c, d} \rightarrow\left(\Lambda\left(s_{i} \nu\right)_{i, 0}\right)^{*} \cap \Lambda\left(s_{i} \nu\right)_{i, d}
$$

for a suitable locally closed subset $\Theta(\nu, i)_{c, d} \subseteq \Theta(\nu, i)$.

Now let $\Xi$ be the set of all tuples $\left(\left(L_{a}\right),\left(M_{a}\right),\left(N_{a}\right), f, g, h\right)$ such that

$$
\left(L_{a}\right) \in \Lambda(\mu)_{i, 0}^{\times}, \quad\left(M_{a}\right) \in\left(\Lambda(\nu)_{i, c}^{\times}\right)^{*}, \quad\left(N_{a}\right) \in \Lambda\left(s_{i} \nu\right)_{i, d}^{\times}
$$

and that $f: M \rightarrow L, \quad g: L \hookrightarrow N$ and $h: N \stackrel{\simeq}{\longrightarrow} \Sigma_{i} M$ are morphisms of $\Pi(Q)$ modules, subject to the condition that $h g f: M \rightarrow \Sigma_{i} M$ is the canonical map from Remark 2.4 (ii).

We inscribe (5.4) as the right column in

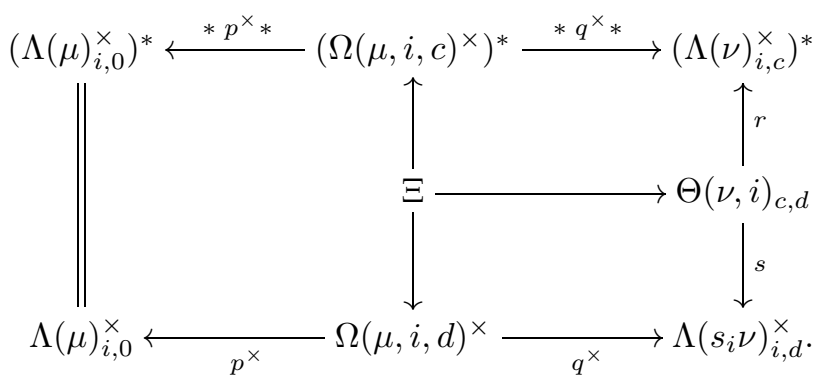

In this commutative diagram, the arrows are the relevant projections and the top and bottom rows are diagrams of the form (5.2). Routine arguments show that the three arrows starting from $\Xi$ are principal bundles, with structural groups $G\left(s_{i} \nu\right)$, $G(\mu)$ and $G(\nu)$. Therefore all the maps on this diagram are locally trivial fibrations with a smooth and connected fiber. They thus induce bijections between the sets of irreducible components.

Now take $b \in B(-\infty)$ as in the statement of the theorem. Let $c=\varphi_{i}\left(b^{*}\right)$, $d=\varepsilon_{i}\left(b^{*}\right)$ and $\mu=\operatorname{wt}\left(\left(\tilde{f}_{i}^{*}\right)^{\max } b\right)$; then the weight of $b$ is $\nu=\mu+c \alpha_{i}$. The remark at the end of section 5.1 says that $d=c-\left\langle\alpha_{i}, \nu\right\rangle$ is non-negative and that $\varphi_{i}\left(\left(\tilde{f}_{i}^{*}\right)^{\max } b\right)=0$, which implies

$$
d=\varphi_{i}\left(S_{i} b\right) \text { and } c+d=2 c-\left\langle\alpha_{i}, \mu+c \alpha_{i}\right\rangle=-\left\langle\alpha_{i}, \mu\right\rangle .
$$

This remark also says that $\varphi_{i}\left(\left(S_{i} b\right)^{*}\right)=0$. The commutativity of our big diagram implies that $\Lambda_{b} \cap\left(\Lambda(\nu)_{i, c}^{\times}\right)^{*}$ and $\Lambda_{S_{i} b} \cap \Lambda\left(s_{i} \nu\right)_{i, d}^{\times}$correspond in the bijection defined by (5.4). We conclude by taking closures in $\Lambda(\nu)_{i, 0}$ and $\left(\Lambda\left(s_{i} \nu\right)_{i, 0}\right)^{*}$. 
5.3. Reformulations. In this subsection, we rewrite in a more direct way the constructions presented in sections 5.1 and 5.2 .

Given a dimension vector $\nu \in Q_{+}$, any point $\left(M_{a}\right) \in \Lambda(\nu)$ can be viewed as a $\Pi(Q)$-module with dimension-vector $\nu$. Conversely, if $M$ is a $\Pi(Q)$-module with dimension-vector $\nu$, then its isomorphism class can be viewed as a $G(\nu)$-orbit in $\Lambda(\nu)$; this orbit will be denoted by $[M]$. $\tilde{f}_{i}^{\max }$.

We now fix $\nu \in Q_{+}$and $i \in I$. Our first reformulation deals with the operation

Proposition 5.4. Let $Z$ be an irreducible component of $\Lambda(\nu)$ and set $c=\varphi_{i}(Z)$ and $\nu^{\prime}=\nu-c \alpha_{i}$. Let $V$ be a dense, open, and $G\left(\nu^{\prime}\right)$-invariant subset of $\tilde{f}_{i}^{\max } Z$. Then $\left\{M \in Z \mid\left[\Sigma_{i}^{*} \Sigma_{i} M\right] \subseteq V\right\}$ contains a dense open subset in $Z$.

Proof. Let $c \in \mathbb{N}$ and set $\nu^{\prime}=\nu-c \alpha_{i}$. The proof of Proposition 2.5) (ii) tells us that if $M$ is a $\Pi(Q)$-module with dimension-vector $\nu$ with an $i$-head of dimension $c$, then $\Sigma_{i}^{*} \Sigma_{i} M$ identifies canonically with the unique submodule of $M$ with dimensionvector $\nu^{\prime}$. With the notation of section [5.1, this means that $\Omega\left(\nu^{\prime}, i, c\right)$ is the set of triples $\left(\left(N_{a}\right),\left(M_{a}\right), g\right)$ such that $\left(N_{a}\right) \in \Lambda\left(\nu^{\prime}\right)_{i, 0},\left(M_{a}\right) \in \Lambda(\nu)_{i, c}$ and $g: N \rightarrow$ $\Sigma_{i}^{*} \Sigma_{i} M$ is an isomorphism of $\Pi(Q)$-modules. Looking at the diagram (5.1), we thus see that for each $\left(M_{a}\right) \in \Lambda(\nu)_{i, c}$, the set $p\left(q^{-1}(M)\right)$ is the orbit $\left[\Sigma_{i}^{*} \Sigma_{i} M\right]$.

Now let $Z$ be an irreducible component of $\Lambda(\nu)$. Put $c=\varphi_{i}(Z)$ in the discussion just above; thus $U=Z \cap \Lambda(\nu)_{i, c}$ is a dense open subset of $Z$. By definition of the operation $\tilde{f}_{i}^{\max }$, the diagram (5.1) restricts to

$$
U^{\prime} \stackrel{p}{\leftarrow} \Omega^{\prime} \stackrel{q}{\rightarrow} U,
$$

where $\Omega^{\prime}$ is an irreducible component of $\Omega\left(\nu^{\prime}, i, c\right)$ and $U^{\prime}=\left(\tilde{f}_{i}^{\max } Z\right) \cap \Lambda\left(\nu^{\prime}\right)_{i, 0}$ is dense open in $\tilde{f}_{i}^{\max } Z$. From the fact that $U^{\prime} \cap V$ is open dense in $U^{\prime}$, it follows that $q\left(p^{-1}\left(U^{\prime} \cap V\right)\right)$ is open dense in $U$.

To conclude the proof, it now suffices to observe that

$$
\begin{aligned}
\left\{M \in Z \mid\left[\Sigma_{i}^{*} \Sigma_{i} M\right] \subseteq V\right\} & =\left\{M \in Z \mid\left[\Sigma_{i}^{*} \Sigma_{i} M\right] \text { meets } V\right\} \\
& \supseteq\left\{M \in U \mid p\left(q^{-1}(M)\right) \text { meets } U^{\prime} \cap V\right\} \\
& =q\left(p^{-1}\left(U^{\prime} \cap V\right)\right) ;
\end{aligned}
$$

in this computation, the first equality comes from the $G\left(\nu^{\prime}\right)$-invariance of $V$.

Our second reformulation concerns the operation $S_{i}$.

Proposition 5.5. Let $b \in B(-\infty)$ and set

$$
\nu=\operatorname{wt}(b), \quad c=\varphi_{i}(b), \quad U=\Lambda_{b} \cap \Lambda(\nu)_{i, c}, \quad b^{\prime}=S_{i}\left(\tilde{f}_{i}^{\max } b\right) \quad \text { and } \quad \nu^{\prime}=\operatorname{wt}\left(b^{\prime}\right) .
$$

Let $V$ be an open, dense and $G\left(\nu^{\prime}\right)$-invariant subset of $\Lambda_{b^{\prime}}$. Then $\left\{\left(M_{a}\right) \in U\right.$ | $\left.\left[\Sigma_{i} M\right] \subseteq V\right\}$ contains a dense open subset of $U$.

Proof. The proof of the particular case $c=0$ is completely analogous to the proof of Proposition 5.4. Indeed, we first note that $b^{\prime}=S_{i} b$, hence $\nu^{\prime}=s_{i} \nu$, and then we let the diagram (5.3) play the role of the diagram (5.1) in our previous discussion, using Theorem 5.3 to identify the bijection between the sets of irreducible components.

The general case is a combination of this particular case $c=0$ with the situation studied in Proposition 5.4. This is obvious on the crystal side; on the side of $\Pi(Q)$ modules, one uses the equality $\Sigma_{i} M \cong \Sigma_{i}\left(\Sigma_{i}^{*} \Sigma_{i} M\right)$ noticed in section 2.2 . 


\section{MV POLYTOPES}

In section 4.3, to each $\Pi(Q)$-module $T$, we associated the pseudo-Weyl polytope $\operatorname{Pol}(T)$ with hyperplane datum $\left(D_{\gamma}(T)\right)_{\gamma \in \Gamma}$. MV polytopes are special pseudoWeyl polytopes, defined by certain rank 2 conditions. The work of Anderson [1] and the second author 13 establishes a link between these MV polytopes and the combinatorics of representations. In particular, MV polytopes form a model for the crystal $B(-\infty)$.

Our goal in this section is to show that if $T$ is a general point in a component $Z \subseteq \Lambda(\nu)$, then the polytope $\operatorname{Pol}(T)$ constructed in section 4.3 is an MV polytope. Moreover, we show that this construction provides an isomorphism of crystals between $\mathbf{B}$ and the set of MV polytopes.

6.1. Tropical Plücker relations. A hexagon is a triple $(w, i, j) \in W \times I^{2}$ such that $a_{i j}=-1, \ell\left(w s_{i}\right)>\ell(w)$ and $\ell\left(w s_{j}\right)>\ell(w)$. (This terminology comes from the fact that such a triple corresponds to an hexagonal 2-face of any pseudo-Weyl polytope.)

We say that a collection of real numbers $\left(A_{\gamma}\right)_{\gamma \in \Gamma}$ satisfies the tropical Plücker relation at the hexagon $(w, i, j)$ if

$$
A_{-w s_{i} \omega_{i}}+A_{-w s_{j} \omega_{j}}=\max \left(A_{-w \omega_{i}}+A_{-w s_{i} s_{j} \omega_{j}}, A_{-w s_{j} s_{i} \omega_{i}}+A_{-w \omega_{j}}\right) .
$$

(The minus signs here are not essential, for they can be removed by replacing $w$ by $w w_{0}$. They have been inserted to simplify the statement of Lemma 6.2.)

We say that a collection $\left(A_{\gamma}\right)_{\gamma \in \Gamma}$ is a BZ datum if the following three conditions hold:

(BZ1) Each $A_{\gamma}$ is an integer and each $A_{-\omega_{i}}$ is zero.

(BZ2) All edge inequalities (4.5) are satisfied.

(BZ3) All possible tropical Plücker relations are satisfied.

We say that a pseudo-Weyl polytope is an MV polytope if its hyperplane datum is a BZ datum. Thus there is a canonical bijection between the set of MV polytopes and the set of BZ data.

For any $\Pi(Q)$-module $T$, the collection $\left(D_{\gamma}(T)\right)_{\gamma \in \Gamma}$ obviously satisfies (BZ1). Proposition 4.2 says that it also satisfies (BZ2); this fact was indeed used in section 4.3 to associate to $T$ its pseudo-Weyl polytope $\operatorname{Pol}(T)$. We will now prove that when $T$ is the general point in a component of a variety $\Lambda(\nu)$, then $\left(D_{\gamma}(T)\right)_{\gamma \in \Gamma}$ satisfies (BZ3); in other words, $\mathrm{Pol}(T)$ is an MV polytope.

More precisely, note that $D_{\gamma}$ is a constructible function on $\Lambda(\nu)$. Hence any irreducible component $Z$ of $\Lambda(\nu)$ contains a dense open subset on which $D_{\gamma}$ takes a constant value: we denote this value by $D_{\gamma}(Z)$. There is thus an open and dense subset $\Omega_{Z} \subseteq Z$ such that each point $T \in \Omega_{Z}$ satisfies $D_{\gamma}(T)=D_{\gamma}(Z)$ for each $\gamma \in \Gamma$ (and hence for each $\gamma \in P$ ); and one may even demand that $\Omega_{Z}$ be $G(\nu)$-invariant, for each function $D_{\gamma}$ is $G(\nu)$-invariant.

Theorem 6.1. Let $\nu \in \mathbb{N}^{I}$ be a dimension-vector. Then for any irreducible component $Z$ of $\Lambda(\nu)$, the collection $\left(D_{\gamma}(Z)\right)_{\gamma \in \Gamma}$ satisfies the tropical Plücker relations.

We have to prove a relation at each hexagon $(w, i, j)$. We will proceed by induction on $\ell(w)$. We begin by checking the base case $w=1$. 
Lemma 6.2. Let $(i, j)$ be a pair of vertices in the Dynkin diagram, connected to each other. Let $\nu$ be a dimension-vector and $Z$ be an irreducible component of $\Lambda(\nu)$. Then $\left(D_{\gamma}(Z)\right)$ satisfies the tropical Plücker relation at $(1, i, j)$.

Proof. Let $i, j$ and $Z$ be as in the statement of the lemma. Theorem 3.1 (ii) implies that $N\left(-s_{i} \omega_{i}\right) \cong S_{i}$ and $N\left(-s_{j} \omega_{j}\right) \cong S_{j}$. Let us set $T_{i}=N\left(-s_{j} s_{i} \omega_{i}\right)$ and $T_{j}=N\left(-s_{i} s_{j} \omega_{j}\right)$. We have $\operatorname{soc} T_{i} \cong S_{i}$ and $\operatorname{soc} T_{j} \cong S_{j}$, and both $T_{i}$ and $T_{j}$ have dimension-vector $\alpha_{i}+\alpha_{j}$, so these modules are represented by

$$
T_{i}: \swarrow^{j} \text { and } T_{j}:{ }^{i} \searrow_{j}
$$

We want to show that for a general point $M$ in $Z$,

$\operatorname{dim} \operatorname{Hom}\left(S_{i}, M\right)+\operatorname{dim} \operatorname{Hom}\left(S_{j}, M\right)=\max \left(\operatorname{dim} \operatorname{Hom}\left(T_{i}, M\right), \operatorname{dim} \operatorname{Hom}\left(T_{j}, M\right)\right)$.

So let us take a point $M$ in $\Omega_{Z}$, and let us adopt the same local representation for $M$ as the one used in the proof of Proposition 2.7 we abbreviate the part

$$
\bigoplus_{\substack{a \in H \\ t(a)=i \\ a \neq c^{*}}} M_{s(a)} \stackrel{\left(\varepsilon(a) M_{a}\right)}{\rightleftarrows\left(M_{a^{*}}\right)} M_{i} \underset{M_{c^{*}}}{\stackrel{M_{c}}{\rightleftarrows}} M_{j} \underset{\left(\varepsilon(b) M_{b}\right)}{\stackrel{\left(M_{b^{*}}\right)}{\rightleftarrows}} \bigoplus_{\substack{b \in H \\ t(b)=j \\ b \neq c}} M_{s(b)}
$$

of the datum of $M$ by the notation

$$
R \underset{l}{\stackrel{k}{\rightleftarrows}} V \underset{g}{\stackrel{f}{\rightleftarrows}} W \underset{n}{\stackrel{m}{\rightleftarrows}} S .
$$

The preprojective relations at $i$ and $j$ then read

$$
k l+\varepsilon\left(c^{*}\right) g f=0 \quad \text { and } \quad n m+\varepsilon(c) f g=0 .
$$

We are interested in the dimension of the spaces

$$
\begin{aligned}
& \operatorname{Hom}\left(S_{i}, M\right) \cong \operatorname{soc}_{i} M=\operatorname{ker} f \cap \operatorname{ker} l, \\
& \operatorname{Hom}\left(S_{j}, M\right) \cong \operatorname{soc}_{j} M=\operatorname{ker} g \cap \operatorname{ker} m, \\
& \operatorname{Hom}\left(T_{i}, M\right) \cong \operatorname{ker} m \cap g^{-1}\left(\operatorname{soc}_{i} M\right)=\operatorname{ker} m \cap g^{-1}(\operatorname{ker} f \cap \operatorname{ker} l), \\
& \operatorname{Hom}\left(T_{j}, M\right) \cong \operatorname{ker} l \cap f^{-1}\left(\operatorname{soc}_{j} M\right)=\operatorname{ker} l \cap f^{-1}(\operatorname{ker} g \cap \operatorname{ker} m) .
\end{aligned}
$$

By the preprojective relations, $\operatorname{ker} m \subseteq g^{-1}(\operatorname{ker} f)$, so $\operatorname{Hom}\left(T_{i}, M\right) \cong \operatorname{ker} m \cap$ $g^{-1}($ ker $l)$. Hence

$\frac{\operatorname{Hom}\left(T_{i}, M\right)}{\operatorname{Hom}\left(S_{j}, M\right)} \cong \frac{\operatorname{ker} m \cap g^{-1}(\operatorname{ker} l)}{\operatorname{ker} m \cap \operatorname{ker} g} \cong g(\operatorname{ker} m) \cap \operatorname{ker} l \subseteq \operatorname{ker} f \cap \operatorname{ker} l \cong \operatorname{Hom}\left(S_{i}, M\right)$, and so we see that

$$
\operatorname{dim} \operatorname{Hom}\left(S_{i}, M\right)+\operatorname{dim} \operatorname{Hom}\left(S_{j}, M\right) \geq \operatorname{dim} \operatorname{Hom}\left(T_{i}, M\right)
$$

with equality if and only if the inclusion

$$
g(\operatorname{ker} m) \cap \operatorname{ker} l \subseteq \operatorname{ker} f \cap \operatorname{ker} l
$$

is an equality. Likewise,

$$
\operatorname{dim} \operatorname{Hom}\left(S_{i}, M\right)+\operatorname{dim} \operatorname{Hom}\left(S_{j}, M\right) \geq \operatorname{dim} \operatorname{Hom}\left(T_{j}, M\right)
$$


with equality if and only if the inclusion

$$
f(\operatorname{ker} l) \cap \operatorname{ker} m \subseteq \operatorname{ker} g \cap \operatorname{ker} m
$$

is an equality.

At this point, it remains to show that the inclusions $(\dagger)$ and $(\ddagger)$ cannot be both strict. We proceed by way of contradiction and assume that there are $v \in(\operatorname{ker} f \cap \operatorname{ker} l) \backslash(g(\operatorname{ker} m) \cap \operatorname{ker} l) \quad$ and $\quad w \in(\operatorname{ker} g \cap \operatorname{ker} m) \backslash(f(\operatorname{ker} l) \cap \operatorname{ker} m)$. We will construct a one-dimensional family $\left(M_{t}\right)$ of points in $\Lambda(\nu)$ such that $M_{0}=$ $M$ and

$$
D_{-s_{j} \omega_{j}}\left(M_{t}\right)=\operatorname{dim} \operatorname{Hom}\left(S_{j}, M_{t}\right) \neq \operatorname{dim} \operatorname{Hom}\left(S_{j}, M\right)=D_{-s_{j} \omega_{j}}(M)=D_{-s_{j} \omega_{j}}(Z)
$$

for all $t \neq 0$. Thus $M_{t}$ cannot be in $\Omega_{Z}$ for $t \neq 0$, which contradicts the fact that $M$ was chosen in the open set $\Omega_{Z}$.

We distinguish two cases. Suppose first that $w \notin \operatorname{im} f$. Then we can choose $W_{1} \subseteq W$ complementary to $\operatorname{span}_{K}(w)$ and such that $\operatorname{im} f \subseteq W_{1}$. For each $t \in K$, we define $M_{t}$ to be the same as $M$ except that the linear map $g$ is deformed to $g_{t}$ defined as follows:

$$
\left.g_{t}\right|_{W_{1}}=\left.g\right|_{W_{1}}, \quad g_{t}(w)=t v .
$$

The preprojective relations are still satisfied, so that $M_{t} \in \Lambda(\nu)$. On the other hand, for all $t \neq 0$,

ker $g_{t} \cap \operatorname{ker} m \subsetneq \operatorname{ker} g \cap \operatorname{ker} m, \quad$ hence $\quad \operatorname{dim} \operatorname{Hom}\left(S_{j}, M_{t}\right)<\operatorname{dim} \operatorname{Hom}\left(S_{j}, M\right)$.

Now suppose that $w \in \operatorname{im} f$ and choose $u \in f^{-1}(w)$. Choose a complement $W_{1}$ to $\operatorname{span}_{K}(w)$ inside $W$ such that $f(\operatorname{ker} l) \subseteq W_{1}$. Since $w \in \operatorname{ker} g$, we have $g f(u)=0$ and so $k l(u)=0$. Let $x=l(u) \in R$. Note that $x \notin l\left(f^{-1}\left(W_{1}\right)\right)$ since $w \notin W_{1}+f(\operatorname{ker} l)$. Hence we can find a complementary subspace $R_{1}$ to $\operatorname{span}_{K}(x)$ inside $R$ such that $l\left(f^{-1}\left(W_{1}\right)\right) \subseteq R_{1}$. For each $t \in K$, we define a point $M_{t}$ by setting

$$
\left.g_{t}\right|_{W_{1}}=\left.g\right|_{W_{1}}, \quad g_{t}(w)=t v,\left.\quad k_{t}\right|_{R_{1}}=\left.k\right|_{R_{1}}, \quad k_{t}(x)=\varepsilon(c) t v
$$

and leaving all the other arrows unchanged. It is easy to verify the preprojective relations for $M_{t}$ and to check that again, for all $t \neq 0$,

ker $g_{t} \cap \operatorname{ker} m \subsetneq \operatorname{ker} g \cap \operatorname{ker} m, \quad$ hence $\quad \operatorname{dim} \operatorname{Hom}\left(S_{j}, M_{t}\right)<\operatorname{dim} \operatorname{Hom}\left(S_{j}, M\right)$.

And now we complete the induction.

Proof of Theorem 6.1. Fix a pair $(i, j)$ of connected vertices. We will show, by induction on $\ell(w)$, that the tropical Plücker relation holds at the hexagon $(w, i, j)$ for any $Z$.

The case $w=1$ was taken care of in Lemma 6.2, so let $w \neq 1$, let $\nu$ be a dimension-vector, and let $Z$ be an irreducible component of $\Lambda(\nu)$.

We can find $k \in I$ such that $\ell\left(s_{k} w\right)<\ell(w)$. We can find $b \in B(-\infty)$ such that $Z=\Lambda_{b}$. Set

$$
c=\varphi_{k}\left(b^{*}\right), \quad b^{\prime}=\left(S_{k}\left(\tilde{f}_{k}^{\max } b^{*}\right)\right)^{*} \quad \text { and } \quad Z^{\prime}=\Lambda_{b^{\prime}} .
$$

By Proposition 5.5, the set $\left\{\left(M_{a}\right) \in Z \cap\left(\Lambda(\nu)_{k, c}\right)^{*} \mid\left[\Sigma_{k}^{*} M\right] \subseteq \Omega_{Z^{\prime}}\right\}$ contains a dense open subset of $Z$. We can thus find $M$ that belongs to both this set and 
$\Omega_{Z}$. In particular, we have $D_{s_{k} \gamma}(M)=D_{s_{k} \gamma}(Z)$ and $D_{\gamma}\left(\Sigma_{k}^{*} M\right)=D_{\gamma}\left(Z^{\prime}\right)$ for each chamber weight $\gamma$. Combining this with equation (4.2), we obtain

$$
D_{s_{k} \gamma}(Z)=D_{\gamma}\left(Z^{\prime}\right)-c\left\langle\gamma, \alpha_{k}\right\rangle
$$

whenever $\left\langle\gamma, \alpha_{k}\right\rangle \leq 0$.

Since $\ell\left(s_{k} w s_{i}\right)=\ell\left(s_{k} w\right) \pm 1=\ell(w)-1 \pm 1$ and $\ell\left(s_{k} w s_{i}\right)=\ell\left(w s_{i}\right) \pm 1=\ell(w)+1 \pm 1$, we see that $\ell\left(s_{k} w s_{i}\right)=\ell(w)=\ell\left(s_{k} w\right)+1$. Combined with a similar analysis for $s_{k} w s_{j}$, this shows that $\left(s_{k} w, i, j\right)$ is an hexagon. The chamber weights involved in the tropical Plücker relation for $(w, i, j)$ are of the form $s_{k} \gamma$, where $\gamma$ is a weight involved in the tropical Plücker relation for $\left(s_{k} w, i, j\right)$. Such a weight $\gamma$ is an element in the list

$$
-s_{k} w \omega_{i},-s_{k} w \omega_{j},-s_{k} w s_{i} \omega_{i},-s_{k} w s_{j} \omega_{j},-s_{k} w s_{j} s_{i} \omega_{i},-s_{k} w s_{i} s_{j} \omega_{j},
$$

so has the form $-s_{k} u \omega_{l}$ with $\ell\left(s_{k} u\right)<\ell(u)$; it follows that $\left\langle\gamma, \alpha_{k}\right\rangle \leq 0$, and thus (6.1) holds. From this observation, a simple computation shows that the tropical Plücker relation for $Z$ at the hexagon $(w, i, j)$ is equivalent to the tropical relation for $Z^{\prime}$ at the hexagon $\left(s_{k} w, i, j\right)$. By the inductive hypothesis, the latter holds, hence the former also holds. This concludes the proof.

6.2. MV polytopes and crystal operations. Let $\mathcal{M V}$ be the set of all $\mathrm{MV}$ polytopes. Theorem 7.2 in [13] provides a natural bijection $b \mapsto P(b)$ from $B(-\infty)$ onto $\mathcal{M V}$. Despite appearences, the proof does not fundamentally relies on constructions in the affine Grassmannian; a short recapitulation of the arguments is presented below in Remark 7.7. Under this bijection, $\mathcal{M V}$ inherits from $B(-\infty)$ a crystal structure. This structure is described in section 3.6 in [12] we now recall how it works.

Let $P \in \mathcal{M V}$ with vertex datum $\left(\mu_{w}\right)_{w \in W}$ and hyperplane datum $\left(A_{\gamma}\right)_{\gamma \in \Gamma}$. Then the weight of $P$ is $\operatorname{wt}(P)=\mu_{w_{0}}$. Fix now $i \in I$. As explained in section 4.2 , the length of the edge between the vertices $\mu_{w_{0}}$ and $\mu_{s_{i} w_{0}}$, that is, the number $c$ such that $\mu_{w_{0}}-\mu_{s_{i} w_{0}}=c \alpha_{i}$, is the integer

$$
c=A_{\omega_{i}}+A_{s_{i} \omega_{i}}+\sum_{\substack{j \in I \\ j \neq i}} a_{i j} A_{\omega_{j}} .
$$

We can then define $\varphi_{i}(P)=c$ and $\varepsilon_{i}(P)=c-\left\langle\alpha_{i}\right.$, wt $\left.(P)\right\rangle$.

The key to understand the operators $\tilde{e}_{i}$ and $\tilde{f}_{i}$ is the observation that when the $A_{\gamma}$ are known for all $\gamma$ in

$$
\left\{\omega_{i} \mid i \in I\right\} \cup\left\{\gamma \in \Gamma \mid\left\langle\gamma, \alpha_{i}\right\rangle \leq 0\right\},
$$

then the tropical Plücker relations determine the remaining $A_{\gamma}$. Therefore the demand that the operators $\tilde{e}_{i}$ and $\tilde{f}_{i}$ do not change the $A_{\gamma}$ such that $\left\langle\gamma, \alpha_{i}\right\rangle \leq 0$ and the conditions

$$
\operatorname{wt}\left(\tilde{e}_{i} P\right)=\operatorname{wt}(P)+\alpha_{i} \text { and } \operatorname{wt}\left(\tilde{f}_{i} P\right)=\operatorname{wt}(P)-\alpha_{i}
$$

fully specify $\tilde{e}_{i} P$ and $\tilde{f}_{i} P$. Since the crystal $\mathcal{M V}$ is isomorphic to $B(-\infty)$, the polytope $\tilde{e}_{i} P$ always exists, and the polytope $\tilde{f}_{i} P$ exists if and only if $\varphi_{i}(P)>0$.

By Theorem 6.1 to each component $Z \in \operatorname{Irr} \Lambda(\nu)$ is associated an MV polytope $\operatorname{Pol}(Z)$, defined by the BZ datum $\left(D_{\gamma}(Z)\right)_{\gamma \in \Gamma}$. So we have a map Pol from $\mathbf{B}=$ $\bigsqcup_{\nu \in Q_{+}} \operatorname{Irr} \Lambda(\nu)$ to $\mathcal{M V}$. 
Theorem 6.3. In the triangle below, each arrow is an isomorphism of crystals.

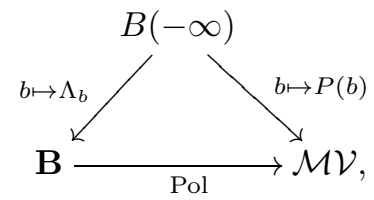

The triangle commutes.

Proof. It is enough to prove that the horizontal arrow is a morphism of crystals, since the two diagonal arrows are isomorphism of crystals and the identity is the only endomorphism of the crystal $B(-\infty)$. By definition, a map of sets is a morphism of crystals if it preserves the five operations wt, $\varepsilon_{i}, \varphi_{i}, \tilde{e}_{i}, \tilde{f}_{i}$. The verification for $\varepsilon_{i}$ is, however, unnecessary, since $\varepsilon_{i}=\varphi_{i}-\left\langle\alpha_{i}\right.$, wt(?) $\rangle$. Noting that

$$
b^{\prime}=\tilde{f}_{i} b \Longleftrightarrow b=\tilde{e}_{i} b^{\prime} \Longleftrightarrow\left(\operatorname{wt}(b)=\operatorname{wt}\left(b^{\prime}\right)+\alpha_{i} \quad \text { and } \quad \tilde{f}_{i}^{\max } b=\tilde{f}_{i}^{\max } b^{\prime}\right),
$$

we are reduced to show that Pol preserves the weight map wt, the functions $\varphi_{i}$ and the operators $\tilde{f}_{i}^{\max }$. Let $Z$ be an element of $\mathbf{B}$.

We have seen in section 4.3 that for any $\Pi(Q)$-module $T$, the vertex $\mu_{w_{0}}(T)$ of $\operatorname{Pol}(T)$ is equal to the dimension-vector of $T$. Taking $T$ in $\Omega_{Z}$, we see that Pol preserves the weight map.

Let $i \in I$. For any $\Pi(Q)$-module $T$, we compute, using Proposition 4.3 at the fourth step and Remark 4.4 (i) at the fifth:

$$
\begin{aligned}
{\operatorname{dim} \operatorname{hd}_{i} T}_{i} & =\operatorname{dim}_{\operatorname{Hom}_{\Pi(Q)}}\left(T, S_{i}\right) \\
& =\operatorname{dim} \operatorname{Hom}_{\Pi(Q)}\left(S_{i}, T^{*}\right) \\
& =D_{-s_{i} \omega_{i}}\left(T^{*}\right) \\
& =D_{s_{i} \omega_{i}}(T)-\left\langle s_{i} \omega_{i}, \underline{\operatorname{dim}} T\right\rangle \\
& =D_{s_{i} \omega_{i}}(T)+D_{\omega_{i}}(T)+\sum_{\substack{j \in I \\
j \neq i}} a_{i j} D_{\omega_{j}}(T) .
\end{aligned}
$$

Hence the function $T \mapsto \operatorname{dim}_{i} T$ is constant on $\Omega_{Z}$, with value

$$
D_{s_{i} \omega_{i}}(Z)+D_{\omega_{i}}(Z)+\sum_{\substack{j \in I \\ j \neq i}} a_{i j} D_{\omega_{j}}(Z) .
$$

This number is thus the general value on $Z$ of that function, so is equal to $\varphi_{i}(Z)$, by definition of the crystal structure of $\mathbf{B}$. On the other hand, this expression is precisely $\varphi_{i}(\operatorname{Pol}(Z))$, by definition of the crystal structure on $\mathcal{M V}$. Therefore Pol preserves the function $\varphi_{i}$.

Again, let $i \in I$, and set $Z^{\prime}=\tilde{f}_{i}^{\max } Z$. Since Pol preserves the weight and $\varphi_{i}$, the equation $\operatorname{wt}\left(Z^{\prime}\right)=\operatorname{wt}(Z)-\varphi_{i}(Z) \alpha_{i}$ translates to

$$
\mathrm{wt}\left(\operatorname{Pol}\left(Z^{\prime}\right)\right)=\operatorname{wt}(\operatorname{Pol}(Z))-\varphi_{i}(\operatorname{Pol}(Z)) \alpha_{i}=\operatorname{wt}\left(\tilde{f}_{i}^{\max } \operatorname{Pol}(Z)\right) .
$$

By Proposition [5.4, the set $\left\{\left(M_{a}\right) \in Z \mid\left[\Sigma_{i}^{*} \Sigma_{i} M\right] \subseteq \Omega_{Z^{\prime}}\right\}$ is open and dense in $Z$. We can thus find an $M$ that belongs to both this set and $\Omega_{Z}$. In particular, we have $D_{\gamma}(M)=D_{\gamma}(Z)$ and $D_{\gamma}\left(\Sigma_{i}^{*} \Sigma_{i} M\right)=D_{\gamma}\left(Z^{\prime}\right)$ for each chamber weight $\gamma$. 
Equation (4.3) then tells us that $D_{\gamma}(Z)=D_{\gamma}\left(Z^{\prime}\right)$ whenever $\left\langle\gamma, \alpha_{i}\right\rangle \leq 0$. This condition, together with $\mathrm{wt}\left(\operatorname{Pol}\left(Z^{\prime}\right)\right)=\operatorname{wt}\left(\tilde{f}_{i}^{\max } \operatorname{Pol}(Z)\right)$, gives $\operatorname{Pol}\left(Z^{\prime}\right)=\tilde{f}_{i}^{\max } \operatorname{Pol}(Z)$. Thus Pol preserves the operation $\tilde{f}_{i}^{\max }$.

\section{From $K Q$ TO $\Pi(Q)$ AND BACK}

In this section, we investigate the relations between $K Q$-modules and $\Pi(Q)$ modules. This theme admits several variations: we relate our reflection functors with the classical version for $K Q$-modules due to Bernstein, Gelfand, Ponomarev; we show that (some of) the $\Pi(Q)$-modules $N\left(-w \omega_{j}\right)$ are (very close to being) induced from an indecomposable $K Q$-module; and we determine combinatorially the bijection from the set of $G(\nu)$-orbits in the representation space $\operatorname{Rep}(K Q, \nu)$ to $\operatorname{Irr} \Lambda(\nu)$ that maps an orbit $\mathscr{O}$ to the closure $\overline{T_{\mathscr{O}}^{*}}$ of its conormal bundle.

7.1. Recall on quivers. In this section, $Q=(I, E)$ is an arbitrary quiver, not necessarily of Dynkin type.

The lattice $\mathbb{Z}^{I}$ is then endowed with a nonsymmetric bilinear form defined by

$$
\langle\mu, \nu\rangle_{Q}=\sum_{i \in I} \mu_{i} \nu_{i}-\sum_{a \in E} \mu_{s(h)} \nu_{t(h)}
$$

and called the Euler form. Then the symmetric bilinear form defined in section 2.1 is

$$
\langle\mu, \nu\rangle=\langle\mu, \nu\rangle_{Q}+\langle\nu, \mu\rangle_{Q}
$$

The following formula is well known: if $M$ and $N$ are two $K Q$-modules, then

$$
\langle\underline{\operatorname{dim}} M, \underline{\operatorname{dim}} N\rangle_{Q}=\operatorname{dim} \operatorname{Hom}_{K Q}(M, N)-\operatorname{dim}_{\operatorname{Ext}_{K Q}^{1}}^{1}(M, N) .
$$

Assume that $i$ is a source of $Q$ and let $\sigma_{i} Q$ denote the quiver obtained from $Q$ by replacing any arrow $a \in E$ that terminates at $i$ by the arrow $a^{*}$ with the opposite orientation. In this context, we have the traditional Bernstein-Gelfand-Ponomarev reflection functors defined in [5]:

$$
K Q-\bmod \underset{\Phi_{i}^{+}}{\stackrel{\Phi_{i}^{-}}{\rightleftarrows}} K\left(\sigma_{i} Q\right)-\bmod .
$$

In particular, $\Phi_{i}^{-}(M)$ is defined by replacing the vector space $M_{i}$ by the cokernel of the outgoing maps from vertex $i$ and $\Phi_{i}^{+}(M)$ by replacing $M_{i}$ with the kernel of the incoming maps to vertex $i$. The functors $\Phi_{i}^{-}$and $\Phi_{i}^{+}$enjoy properties similar to the ones stated in Proposition 2.5 for the functors $\Sigma_{i}$ and $\Sigma_{i}^{*}$.

Certainly, $K Q$ can be viewed as a subalgebra of $\Pi(Q)$. By restriction, a $\Pi(Q)$ module $T$ gives rise to a $K Q$-module, which we denote by $\left.T\right|_{Q}$; in other words, we forget the action of the arrows $a \in E^{*}$. Now suppose that $i$ is a source in $Q$. The quiver $\sigma_{i} Q$ also gives rise to a preprojective algebra $\Pi\left(\sigma_{i} Q\right)$; it has the same set $H$ of arrows as $\Pi(Q)$, but with a different function $\varepsilon$. There is an isomorphism $\Upsilon_{i}: \Pi\left(\sigma_{i} Q\right) \rightarrow \Pi(Q)$, which changes any arrow $b$ that terminates at $i$ into $-b$ and which fixes all the other arrows in $H$. We can then pull back a $\Pi(Q)$-module $T$ by $\Upsilon_{i}$ and restrict the $\Pi\left(\sigma_{i} Q\right)$-module $\Upsilon_{i}^{*} T$ thus obtained to $K\left(\sigma_{i} Q\right)$.

Proposition 7.1. Let $T$ be a $\Pi(Q)$-module. Then

$$
\left.\Phi_{i}^{+}\left(\left.\left(\Upsilon_{i}^{*} T\right)\right|_{\sigma_{i} Q}\right) \cong\left(\Sigma_{i} T\right)\right|_{Q} \quad \text { and }\left.\quad \Phi_{i}^{-}\left(\left.T\right|_{Q}\right) \cong\left(\Upsilon_{i}^{*} \Sigma_{i}^{*} T\right)\right|_{\sigma_{i} Q}
$$


Proof. The definition of the functors $\Sigma_{i}$ and $\Sigma_{i}^{*}$ visibly extends Bernstein, Gelfand and Ponomarev's construction.

To conclude this section, we present a lemma also related to reflection functors for the single quiver.

Lemma 7.2. Assume that $i$ is a source in $Q$. Then $\left\langle s_{i} \mu, s_{i} \nu\right\rangle_{Q}=\langle\mu, \nu\rangle_{\sigma_{i} Q}$ for all $\mu, \nu \in \mathbb{Z}^{I}$.

Proof. Using the definition of the Euler form and that $i$ is a source in $Q$, one finds that

$$
\left\langle\mu-\mu_{i} \alpha_{i}, \nu\right\rangle_{Q}=\sum_{\substack{j \in I \\ j \neq i}} \mu_{j} \nu_{j}-\sum_{\substack{a \in E \\ i \notin\{s(a), t(a)\}}} \mu_{s(a)} \nu_{t(a)}=\left\langle\mu, \nu-\nu_{i} \alpha_{i}\right\rangle_{\sigma_{i} Q} .
$$

One then computes

$$
\begin{aligned}
\left\langle s_{i} \mu, \nu\right\rangle_{Q} & =\langle\mu, \nu\rangle_{Q}-\left\langle\alpha_{i}, \mu\right\rangle\left\langle\alpha_{i}, \nu\right\rangle_{Q} \\
& =\langle\mu, \nu\rangle_{Q}-\left(\left\langle\mu, \alpha_{i}\right\rangle_{Q}+\left\langle\alpha_{i}, \mu\right\rangle_{Q}\right)\left\langle\alpha_{i}, \nu\right\rangle_{Q} \\
& =\left\langle\mu-\mu_{i} \alpha_{i}, \nu\right\rangle_{Q}-\left\langle\alpha_{i}, \mu\right\rangle_{Q}\left\langle\alpha_{i}, \nu\right\rangle_{Q} \\
& =\left\langle\mu, \nu-\nu_{i} \alpha_{i}\right\rangle_{\sigma_{i} Q}-\left\langle\mu, \alpha_{i}\right\rangle_{\sigma_{i} Q}\left\langle\nu, \alpha_{i}\right\rangle_{\sigma_{i} Q} \\
& =\langle\mu, \nu\rangle_{\sigma_{i} Q}-\left(\left\langle\alpha_{i}, \nu\right\rangle_{\sigma_{i} Q}+\left\langle\nu, \alpha_{i}\right\rangle_{\sigma_{i} Q}\right)\left\langle\mu, \alpha_{i}\right\rangle_{\sigma_{i} Q} \\
& =\langle\mu, \nu\rangle_{\sigma_{i} Q}-\left\langle\alpha_{i}, \nu\right\rangle\left\langle\mu, \alpha_{i}\right\rangle_{\sigma_{i} Q} \\
& =\left\langle\mu, s_{i} \nu\right\rangle_{\sigma_{i} Q} .
\end{aligned}
$$

This formula is of course equivalent to the one written in the statement.

Remark 7.3. There is a more conceptual proof of this lemma. The dimension-vector defines an isomorphism between $\mathbb{Z}^{I}$ and the Grothendieck group of the derived category $\mathbb{D}^{b}(K Q$-mod $)$, and one has

$$
\sum_{n \in \mathbb{Z}}(-1)^{n} \operatorname{dim} \operatorname{Ext}_{\mathbb{D}^{b}(K(Q))}^{n}(M, N)=\langle\underline{\operatorname{dim}} M, \underline{\operatorname{dim}} N\rangle_{Q}
$$

for any two objects $M$ and $N$ in this category. A similar statement applies to the derived category $\mathbb{D}^{b}\left(K\left(\sigma_{i} Q\right)\right.$-mod). Now the right-exact functor $\Phi_{i}^{+}$induces an equivalence of derived categories

$$
\mathbb{R} \Phi_{i}^{+}: \mathbb{D}^{b}\left(K\left(\sigma_{i} Q\right) \text {-mod }\right) \rightarrow \mathbb{D}^{b}(K Q \text {-mod })
$$

and one has

$$
\underline{\operatorname{dim}} \mathbb{R} \Phi_{i}^{+} M=s_{i}(\underline{\operatorname{dim}} M) .
$$

7.2. Modules $N(\gamma)$ and induction. We now go back to our set-up where $Q$ is obtained by choosing an orientation of the Dynkin diagram $\Gamma$.

We denote the quiver opposite to $Q$ by $Q^{*}$. Recall that a sequence $\left(i_{1}, \ldots, i_{n}\right)$ of vertices is called adapted to $Q^{*}$ if $i_{1}$ is a source of $Q, i_{2}$ is a source of $\sigma_{i_{1}} Q, i_{3}$ is a source of $\sigma_{i_{2}} \sigma_{i_{1}} Q$, and so on (see section 4.7 in [18]).

We first prove a combinatorial version of our induction result.

Proposition 7.4. Assume that the sequence $\left(i_{1}, \ldots, i_{n}\right)$ is adapted to $Q^{*}$ and that $s_{i_{1}} \cdots s_{i_{n}}$ is a reduced expression of an element $w \in W$. Then $\left\langle-w \alpha_{i_{n}}, ?\right\rangle_{Q}=$ $\left\langle-w \omega_{i_{n}}, ?\right\rangle$ as linear forms on $Q$. 
Proof. For each $j \in I$ different from $i_{1}$,

$$
\left\langle\alpha_{i_{1}}-\omega_{i_{1}}, \alpha_{j}\right\rangle=\left\langle\alpha_{i_{1}}, \alpha_{j}\right\rangle=\left\langle\alpha_{i_{1}}, \alpha_{j}\right\rangle_{Q}+\left\langle\alpha_{j}, \alpha_{i_{1}}\right\rangle_{Q}=\left\langle\alpha_{i_{1}}, \alpha_{j}\right\rangle_{Q},
$$

because $i_{1}$ is a source in $Q$. The equality $\left\langle\alpha_{i_{1}}-\omega_{i_{1}}, \alpha_{j}\right\rangle=\left\langle\alpha_{i_{1}}, \alpha_{j}\right\rangle_{Q}$ also holds for $j=i_{1}$. We deduce by linearity that

$$
\left\langle\alpha_{i_{1}}-\omega_{i_{1}}, ?\right\rangle=\left\langle\alpha_{i_{1}}, ?\right\rangle_{Q}
$$

which is the case $n=1$.

For higher value of $n$, we proceed by induction, using Lemma 7.2 and the $W$ invariance of $\langle$,$\rangle .$

We will use the notation $S_{i}$ for the simple $K Q$-module attached to the vertex $i$ and we will also speak of $i$-socle for $K Q$-modules.

Recall that by the classical theorem of Gabriel, for each positive root $\beta$, there is a unique (up to isomorphism) indecomposable $K Q$-module with dimension-vector $\beta$, which we denote by $M(\beta)$. Moreover, Bernstein, Gelfand and Ponomarev [5] showed that these indecomposable modules may be constructed using their reflection functors. Let $Q$ be a quiver and let $i$ be a source in $Q$. Let $\beta$ be a positive root. Then

$$
\Phi_{i}^{-} M(\beta)=\left\{\begin{array}{l}
0 \text { if } \beta=\alpha_{i}, \\
M\left(s_{i} \beta\right) \text { otherwise }
\end{array}\right.
$$

where $M\left(s_{i} \beta\right)$ denotes the indecomposable $K\left(\sigma_{i} Q\right)$ module with dimension-vector $s_{i} \beta$. Starting with the base case $M\left(\alpha_{i}\right)=S_{i}$, this gives a recursive way to construct all $M(\beta)$.

Ringel has observed (see section 4 in [27]) that if $\alpha$ and $\beta$ are two positive roots, then at least one of the two spaces $\operatorname{Hom}_{K Q}(M(\alpha), M(\beta))$ or $\operatorname{Ext}_{K Q}^{1}(M(\alpha), M(\beta))$ is zero. Thus equation (7.1) gives

$$
\left\{\begin{array}{l}
\operatorname{dim} \operatorname{Hom}_{K Q}(M(\alpha), M(\beta))=\max \left(0,\langle\alpha, \beta\rangle_{Q}\right), \\
\operatorname{dim} \operatorname{Ext}_{K Q}^{1}(M(\alpha), M(\beta))=\max \left(0,-\langle\alpha, \beta\rangle_{Q}\right) .
\end{array}\right.
$$

The following proposition is the analogue for the single quiver $Q$ of equation (4.2).

Proposition 7.5. Assume that $i$ is a source in $Q$ and let $\beta$ be a positive root, different from $\alpha_{i}$. Let $T$ be a KQ-module. Then

$\operatorname{dim} \operatorname{Hom}_{K Q}\left(M\left(s_{i} \beta\right), T\right)=\operatorname{dim} \operatorname{Hom}_{K\left(\sigma_{i} Q\right)}\left(M(\beta), \Phi_{i}^{-} T\right)-\left\langle\beta, \underline{\operatorname{dim}} \operatorname{soc}_{i} T\right\rangle_{\sigma_{i} Q}$.

(In this equality, it is implicitly understood that $M\left(s_{i} \beta\right)$ is the indecomposable $K Q$ module with dimension-vector $s_{i} \beta$, and that $M(\beta)$ is the indecomposable $K\left(\sigma_{i} Q\right)$ module with dimension-vector $\beta$.)

Proof. We first notice that $\left\langle s_{i} \beta, \alpha_{i}\right\rangle_{Q} \geq 0$, for $i$ is a source in $Q$ and $s_{i} \beta \in Q_{+}$. Thus

$$
\operatorname{Ext}_{K Q}^{1}\left(M\left(s_{i} \beta\right), S_{i}\right)=0 \quad \text { and } \quad \operatorname{dim} \operatorname{Hom}_{K Q}\left(M\left(s_{i} \beta\right), S_{i}\right)=\left\langle s_{i} \beta, \alpha_{i}\right\rangle_{Q} .
$$

Lemma 7.2. moreover, says that $\left\langle s_{i} \beta, \alpha_{i}\right\rangle_{Q}=-\left\langle\beta, \alpha_{i}\right\rangle_{\sigma_{i} Q}$.

On the other hand, the functor $\Phi_{i}^{-}$maps $M\left(s_{i} \beta\right)$ to $M(\beta)$ by (7.2); by adjunction, we thus get

$$
\begin{aligned}
\operatorname{Hom}_{K\left(\sigma_{i} Q\right)}\left(M(\beta), \Phi_{i}^{-} T\right) & \left.\cong \operatorname{Hom}_{K\left(\sigma_{i} Q\right)}\left(\Phi_{i}^{-} M\left(s_{i} \beta\right), \Phi_{i}^{-} T\right)\right) \\
& \cong \operatorname{Hom}_{K Q}\left(M\left(s_{i} \beta\right), \Phi_{i}^{+} \Phi_{i}^{-} T\right) .
\end{aligned}
$$


It remains to apply the functor $\operatorname{Hom}_{K Q}\left(M\left(s_{i} \beta\right), ?\right)$ to the (split) short exact sequence

$$
0 \rightarrow \operatorname{soc}_{i} T \rightarrow T \rightarrow \Phi_{i}^{+} \Phi_{i}^{-} T \rightarrow 0
$$

and to take dimensions.

We now have all the ingredients to prove the following result.

Theorem 7.6. Assume that the sequence $\left(i_{1}, \ldots, i_{n}\right)$ is adapted to $Q^{*}$ and that $s_{i_{1}} \cdots s_{i_{n}}$ is a reduced expression of an element $w \in W$. Then for any $\Pi(Q)$-module $T$,

$$
\operatorname{dim} \operatorname{Hom}_{K Q}\left(M\left(-w \alpha_{i_{n}}\right),\left.T\right|_{Q}\right)=\operatorname{dim} \operatorname{Hom}_{\Pi(Q)}\left(N\left(-w \omega_{i_{n}}\right), T\right) .
$$

Proof. We first observe that since $i_{1}$ is a source of $Q$, the $i_{1}$-socles of the $\Pi(Q)$ module $T$ and of the $K Q$-module $\left.T\right|_{Q}$ coincide. In particular, they have the same dimension (-vector).

The case $n=1$ is then obvious, because then both $M\left(-w \alpha_{i_{n}}\right)$ and $N\left(-w \omega_{i_{n}}\right)$ are the simple module $S_{i_{1}}$.

In the case $n>1$, we proceed by induction on $n$. More precisely, we assume that the equality holds for the sequence $\left(i_{2}, \ldots, i_{n}\right)$, the quiver $\sigma_{i_{1}} Q$ and the $\Pi\left(\sigma_{i_{1}} Q\right)$ module $\Upsilon_{i_{1}}^{*} \Sigma_{i_{1}}^{*} T$. A straightforward computation based on Propositions 4.1, 7.1, 7.4 and 7.5 then leads to the equality for the sequence $\left(i_{1}, \ldots, i_{n}\right)$, the quiver $Q$ and the $\Pi(Q)$-module $T$.

Theorem 7.6 shows the existence of an isomorphism

$$
\operatorname{Hom}_{K Q}\left(M\left(-w \alpha_{i_{n}}\right),\left.T\right|_{Q}\right) \cong \operatorname{Hom}_{\Pi(Q)}\left(N\left(-w \omega_{i_{n}}\right), T\right) .
$$

We believe that this isomorphism can be made functorial in $T \in \Pi(Q)$-mod; in other words, we believe that $N\left(-w \omega_{i_{n}}\right)$ is isomorphic to $\Pi(Q) \otimes_{K Q} M\left(-w \alpha_{i_{n}}\right)$. This fact can probably be deduced from the work of Geiß, Leclerc and Schröer [10]. Anyway, this would generally not give a description of all the modules $N(\gamma)$ with $\gamma$ a chamber weight; indeed, only in type $A$ can one always write $\gamma=-s_{i_{1}} \cdots s_{i_{n}} \omega_{i_{n}}$ for a sequence $\left(i_{1}, \ldots, i_{n}\right)$ adapted to an orientation of the graph $\Gamma$.

7.3. Irreducible components and conormal bundles. Let $\nu \in Q_{+}$be a dimension-vector. The isomorphism classes of representations of the quiver $Q$ with dimension-vector $\nu$ are the orbits of the group $G(\nu)$ on the representation space $\operatorname{Rep}(K Q, \nu)$. In Proposition 14.2 of [20], Lusztig shows that the irreducible components of $\Lambda(\nu)$ are precisely the closures of the conormal bundles to the $G(\nu)$-orbits in $\operatorname{Rep}(K Q, \nu)$. More precisely, he observes (loc. cit., 12.8 (a)) that under the trace duality

$$
\prod_{a \in H} \operatorname{Hom}_{K}\left(K^{\nu_{s(a)}}, K^{\nu_{t(a)}}\right) \cong \operatorname{Rep}(K Q, \nu) \times \operatorname{Rep}(K Q, \nu)^{*},
$$

any point $\left(M_{a}\right) \in \Lambda(\nu)$ belongs to the conormal bundle $T_{\mathscr{O}}^{*}$, where $\mathscr{O}$ is the $G(\nu)$ orbit in $\operatorname{Rep}(K Q, \nu)$ that corresponds to the isomorphism class of the $K Q$-module $\left.M\right|_{Q}$.

Lusztig's interest in the varieties $\Lambda(\nu)$ arose in connection with its study of the canonical basis $\mathcal{B}$ of the quantum group $U_{q}(\mathfrak{n})$, the positive part of $U_{q}(\mathfrak{g})$. Geometrically, an element $b$ in the canonical basis is represented by a simple $G(\nu)$ equivariant perverse sheaf $L_{b, Q}$ on $\operatorname{Rep}(K Q, \nu)$, where $\nu$ is the weight of $b$, and such a sheaf is always the intersection cohomology sheaf $\operatorname{IC}\left(\overline{\mathscr{O}_{b, Q}}\right)$ (suitably shifted) of 
the closure of a $G(\nu)$-orbit (with coefficients in the trivial local system). The singular support $S S\left(L_{b, Q}\right)$ then contains $\overline{T_{\mathscr{O}_{b, Q}}^{*}}$.

The canonical basis $\mathcal{B}$ has the structure of a crystal, which identifies it with $B(-\infty)$. We can thus change slightly the notation and suppose that the letter $b$ in $L_{b, Q}$ and $\mathscr{O}_{b, Q}$ denotes an element of $B(-\infty)$. In [16], Kashiwara and Saito proved that the labelling of the canonical and semicanonical bases are compatible in the sense that $\Lambda_{b}=\overline{T_{\mathscr{O}_{b, Q}}^{*}}$.

Unfortunately, Kashiwara and Saito's proof contains a small gap, that was later filled by Kimura [17. More precisely, the heart of the proof is the fact that the only weight-preserving permutation $s$ of $B(-\infty)$ such that $\Lambda_{s(b)} \subseteq S S\left(L_{b, \Omega}\right)$ for each $b \in B(-\infty)$ is the identity $([16$, p. $27,1.6)$. This fact holds in full generality (not only in the finite ADE case), but to prove it, one should not rely on the erroneous equation (6.2), but rather on the Lemma 8.2.1. We thank Yoshihisa Saito for detailed explanations on that matter [31.

Our aim in this final section is to apply our results to obtain an alternative proof of the equality $\Lambda_{b}=\overline{T_{\mathscr{O}_{b, Q}}^{*}}$. In other words, we will prove that the map $b \mapsto \overline{T_{\mathscr{O}_{b, Q}}^{*}}$ from $B(-\infty)$ to $\mathbf{B}$ is a morphism of crystal. One difficulty is that this map a priori depends on the choice of the orientation $Q$.

We first need to recall some facts concerning parametrizations of $B(-\infty)$. As before, $w_{0}$ is the longest element in $W$. We denote its length by $N$ and call $\mathscr{X}$ the set of all tuples $\mathbf{i}=\left(i_{1}, \ldots, i_{N}\right)$ such that $s_{i_{1}} \cdots s_{i_{N}}$ is a reduced expression of $w_{0}$. As explained by Lusztig [18, to each $\mathbf{i} \in \mathscr{X}$ corresponds a bijection $\mathbf{n}(?, \mathbf{i}): B(-\infty) \rightarrow$ $\mathbb{N}^{N}$, which is the combinatorial counterpart of the transition matrix between the canonical basis $\mathcal{B}$ and the PBW basis of $U_{q}(\mathfrak{n})$ defined by $\mathbf{i}$. These bijections can be collectively characterized by the properties (L1), (L2a) and (L2b) below. In these statements, $(b, \mathbf{i}) \in B(-\infty) \times \mathscr{X}$ and $\left(n_{1}, \ldots, n_{N}\right)$ is the tuple $\mathbf{n}(b, \mathbf{i})$.

(L1) One has

$$
n_{1}=\varphi_{i_{1}}(b), \quad \mathbf{n}\left(\tilde{e}_{i_{1}} b, \mathbf{i}\right)=\left(n_{1}+1, n_{2}, \ldots, n_{N}\right), \quad \mathbf{n}\left(\tilde{f}_{i_{1}}^{\max } b, \mathbf{i}\right)=\left(0, n_{2}, \ldots, n_{N}\right) .
$$

(L2a) Let $(i, j) \in I^{2}$ and $k \in\{1, \ldots, N-1\}$ be such that $a_{i j}=0$ and $\left(i_{k}, i_{k+1}\right)=(i, j)$. Set $\mathbf{j}=\left(i_{1}, \ldots, i_{k-1}, j, i, i_{k+2}, \ldots, i_{N}\right)$. Then

$$
\mathbf{n}(b, \mathbf{j})=\left(n_{1}, \ldots, n_{k-1}, n_{k+1}, n_{k}, n_{k+2}, \ldots, n_{N}\right) .
$$

(L2b) Let $(i, j) \in I^{2}$ and $k \in\{2, \ldots, N-1\}$ be such that $a_{i j}=-1$ and $\left(i_{k-1}, i_{k}, i_{k+1}\right)=(i, j, i)$. Let $(p, q, r)=\left(n_{k-1}, n_{k}, n_{k+1}\right)$ and set $\mathbf{j}=\left(i_{1}, \ldots, i_{k-2}, j, i, j, i_{k+2}, \ldots, i_{N}\right), q^{\prime}=\min (p, r), p^{\prime}=r+q-q^{\prime}$ and $r^{\prime}=p+q-q^{\prime}$. Then

$$
\mathbf{n}(b, \mathbf{j})=\left(n_{1}, \ldots, n_{k-2}, p^{\prime}, q^{\prime}, r^{\prime}, n_{k+2}, \ldots, n_{N}\right) .
$$

(L3) Let $i_{1}^{*} \in I$ be the element such that $w_{0} \alpha_{i_{1}}=-\alpha_{i_{1}^{*}}$. Suppose $n_{1}=0$ and set $\mathbf{j}=\left(i_{2}, i_{3}, \ldots, i_{N}, i_{1}^{*}\right)$. Then

$$
\mathbf{n}\left(S_{i_{1}}(b), \mathbf{j}\right)=\left(n_{2}, n_{3}, \ldots, n_{N}, 0\right) .
$$

Property (L3) is due to Saito (Proposition 3.4.7 in [29]). The interesting point here is that with the help of (L1) and (L3), one can determine $\mathbf{n}(b, \mathbf{i})$ by applying to $b$ the operations $\varphi_{i}, \tilde{f}_{i}^{\max }$ and $S_{i}$, with $i$ running over the successive terms of the sequence $\mathbf{i}$. This procedure is convenient for us, for these three crystal operations have a clear meaning in the preprojective model, thanks to Propositions 5.4 and 5.5] 
Remark 7.7. In this remark, we explain the origin of the bijection $B(-\infty) \cong \mathcal{M V}$ we used in section 6.2

(i) For $\mathbf{i}, \mathbf{j} \in \mathscr{X}$, we can define a permutation $R_{\mathbf{i}}^{\mathbf{j}}$ of $\mathbb{N}^{N}$ by the condition $R_{\mathbf{i}}^{\mathbf{j}} \circ \mathbf{n}(?, \mathbf{i})=\mathbf{n}(?, \mathbf{j})$. Then $b \mapsto \mathbf{n}(b, ?)$ is a bijection from $B(-\infty)$ onto

$$
\widehat{\mathscr{X}}=\left\{\sigma: \mathscr{X} \rightarrow \mathbb{N}^{N} \mid \forall(\mathbf{i}, \mathbf{j}) \in \mathscr{X}^{2}, \sigma(\mathbf{j})=R_{\mathbf{i}}^{\mathbf{j}}(\sigma(\mathbf{i}))\right\} .
$$

The permutations $R_{\mathbf{i}}^{\mathbf{j}}$ extends in a natural fashion to $\mathbb{Z}^{N}$, for it is given by a piecewise linear formula which also makes sense for signed integers. Let $\widehat{\mathscr{X}}$ be the set defined similarly as $\widehat{\mathscr{X}}$, but with $\mathbb{Z}^{N}$ instead of $\mathbb{N}^{N}$.

(ii) To $\sigma \in \widetilde{\mathscr{X}}$, we associate a vertex datum $\left(\mu_{w}\right)_{w \in W}$ as follows: for each $w \in W$, we can find $\mathbf{i} \in \mathscr{X}$ and $k \in\{0, \ldots, N\}$ such that $w w_{0}=s_{i_{1}} \cdots s_{i_{k}}$, and we set

$\mu_{w}=\sum_{r=k+1}^{N} n_{r} \beta_{r}, \quad$ where $\sigma(\mathbf{i})=\left(n_{1}, \ldots, n_{N}\right) \quad$ and $\quad \beta_{r}=s_{i_{1}} \cdots s_{i_{r-1}} \alpha_{i_{r}}$.

(This weight $\mu_{w}$ depends solely on $\sigma$ and $w$ and not on the choice of i.) We then associate an hyperplane datum $\left(A_{\gamma}\right)_{\gamma \in \Gamma}$ by the equation (4.6). Then the map $\sigma \mapsto\left(A_{\gamma}\right)_{\gamma \in \Gamma}$ is a bijection from $\widetilde{\mathscr{X}}$ onto the set of all families that satisfies the conditions (BZ1) and (BZ3) from section 6.1. This fact is proved by specializing Theorem 4.3 in 4 to the tropical semifield.

(iii) The numbers that appear in the left-hand side of the edge inequalities (4.5) are precisely the components of the tuples $\sigma(\mathbf{i})$. Thus $\left(A_{\gamma}\right)_{\gamma \in \Gamma}$ satisfies (BZ2) if and only if each $\sigma(\mathbf{i})$ belongs to $\mathbb{N}^{N}$. By restriction, we therefore get a bijection from $\widehat{\mathscr{X}}$ onto the set of all BZ data. In the end, we obtain a bijection $B(-\infty) \cong \mathcal{M V}$. To sum up, the MV polytope $P(b)$ associated to an element $b \in B(-\infty)$ packs together in a geometrical way the numerical data $\mathbf{n}(b, \mathbf{i})$ for all possible $\mathbf{i}$.

(iv) Keeping the same notation, we now turn to the crystal structure defined on $\mathcal{M V}$ by transport from $B(-\infty)$. For each $w \in W$ such that $\ell\left(s_{i} w w_{0}\right)<$ $\ell\left(w w_{0}\right)$, we can choose an $\mathbf{i} \in \mathscr{X}$ that starts with $i$ in the construction of (ii) above and we have $k>0$; thus $n_{1}$ does not enter in the equation that expresses $\mu_{w}$, and Property (L1) tells us that the crystal operations $\tilde{e}_{i}$ and $\tilde{f}_{i}$ do not change $\mu_{w}$. In terms of the BZ data $\left(A_{\gamma}\right)_{\gamma \in \Gamma}$, this translates into the fact that the operators $\tilde{e}_{i}$ and $\tilde{f}_{i}$ do not change the $A_{\gamma}$ such that $\left\langle\gamma, \alpha_{i}\right\rangle \leq 0$. We thus recover the criterion we used in section 6.2 .

(v) We can understand Property (L3) from the point of view of Theorem 6.3. In more detail, equations (4.1) and (4.6) together imply the relation

$$
\mu_{w}(T)=s_{i} \mu_{s_{i} w}\left(\Sigma_{i} T\right)
$$

for each $\Pi(Q)$-module $T$ and each $w \in W$ such that $\ell\left(s_{i} w\right)>\ell(w)$. Then (L3) is a direct translation of Proposition 5.5.

We are now ready to prove our result.

Proposition 7.8. The equality $\Lambda_{b}=\overline{T_{\mathscr{O}_{b, Q}}^{*}}$ holds for each $b \in B(-\infty)$.

Proof. Let $\mathbf{i} \in \mathscr{X}$ be adapted to the quiver $Q: i_{1}$ is a $\operatorname{sink}$ of $Q, i_{2}$ is a sink of $\sigma_{i_{1}}^{-1} Q$, and so on. (Such a sequence $\mathbf{i}$ always exists, thanks to Proposition 4.12 (b) in $[18]$ ). 
It is enough to show that two elements $b, b^{\prime} \in B(-\infty)$ such that $\Lambda_{b}=\overline{T_{\mathscr{O}_{b^{\prime}, Q}}^{*}}$ have necessarily the same image under the map $\mathbf{n}(?, \mathbf{i})$. So let us take two such elements and set

$$
\left(n_{1}, \ldots, n_{N}\right)=\mathbf{n}(b, \mathbf{i}) \text { and }\left(n_{1}^{\prime}, \ldots, n_{N}^{\prime}\right)=\mathbf{n}\left(b^{\prime}, \mathbf{i}\right) .
$$

Let $T$ be a general point in $\Lambda_{b}$. In view of Property (L3) above, successive applications of Proposition 5.5 show that

$$
n_{k}=\operatorname{dimhd}_{i_{k}}\left(\Sigma_{i_{k-1}} \cdots \Sigma_{i_{1}} T\right) .
$$

Here we can substitute

$$
\left(\Sigma_{i_{k-1}}\left(\Upsilon_{i_{k-1}}^{-1}\right)^{*}\right) \cdots\left(\Sigma_{i_{1}}\left(\Upsilon_{i_{1}}^{-1}\right)^{*}\right) \cong\left(\left(\Upsilon_{i_{k-1}} \cdots \Upsilon_{i_{1}}\right)^{-1}\right)^{*}\left(\Sigma_{i_{k-1}} \cdots \Sigma_{i_{1}}\right)
$$

to $\Sigma_{i_{k-1}} \cdots \Sigma_{i_{1}}$ without changing the $i_{k}$-head.

On the other hand, for each positive $\operatorname{root} \beta$, let $M(\beta)$ be the indecomposable $K Q$-module with dimension-vector $\beta$, and let $\beta_{1}, \ldots, \beta_{N}$ be the indexation of the positive roots defined by i. By sections $4.15-4.16$ in [18], the fact that $\left.T\right|_{Q} \in \mathscr{O}_{b^{\prime}, Q}$ means

$$
\left.T\right|_{Q} \cong M\left(\beta_{1}\right)^{\oplus n_{1}^{\prime}} \oplus \cdots \oplus M\left(\beta_{N}\right)^{\oplus n_{N}^{\prime}} .
$$

It follows from equation (7.2) that

$$
n_{k}^{\prime}=\operatorname{dimhd} i_{i_{k}}\left(\Phi_{i_{k-1}}^{+} \cdots \Phi_{i_{1}}^{+}\left(\left.T\right|_{Q}\right)\right) .
$$

An appeal to Proposition 7.1 now concludes the proof; one just has to observe that since $i_{k}$ is a sink of the quiver $Q^{\prime}=\sigma_{i_{k-1}}^{-1} \cdots \sigma_{i_{1}}^{-1} Q$, the $i_{k}$-head of the $\Pi\left(Q^{\prime}\right)$ module

$$
\left(\Sigma_{i_{k-1}}\left(\Upsilon_{i_{k-1}}^{-1}\right)^{*}\right) \cdots\left(\Sigma_{i_{1}}\left(\Upsilon_{i_{1}}^{-1}\right)^{*}\right)(T)
$$

is the same as its head as a $K Q^{\prime}$-module.

\section{ACKNOWLEDGMENTS}

The authors heartily thank A. Braverman, C. Geiß, B. Keller, B. Leclerc, C. Sadanand, A. Savage, J. Schröer, and P. Tingley for useful conversations. The authors also thank the referee for his careful reading of this paper.

The first author acknowledges support from ANR project RepRed, ANR-09JCJC-0102-01 and the second author acknowledges support from NSERC and AIM. This work began when both authors were participating in the Combinatorial Representation Theory program at MSRI in 2008. Hence we thank MSRI and the organizers of this program for their hospitality and good working environment.

\section{REFERENCES}

1. J. E. Anderson, A polytope calculus for semisimple groups, Duke Math. J. 116 (2003), 567588. MR1958098(2004a:20047)

2. M. Auslander, I. Reiten, S. O. Smalø, Representation theory of Artin algebras, Cambridge Studies in Advanced Mathematics, vol. 36, Cambridge University Press, 1995. MR.1314422 (96c:16015)

3. P. Baumann, J. Kamnitzer, P. Tingley, Affine Mirković-Vilonen polytopes, in preparation.

4. A. Berenstein, A. Zelevinsky, Total positivity in Schubert varities, Comment. Math. Helv. 72 (1997), 128-166. MR1456321 (99g:14064)

5. I. N. Bernstein, I. M. Gelfand, V. A. Ponomarev, Coxeter functors and Gabriel's theorem, Uspehi Mat. Nauk 28 (1973), 19-33. MR0393065 (52:13876)

6. A. B. Buan, O. Iyama, I. Reiten, J. Scott, Cluster structures for 2-Calabi-Yau categories and unipotent groups, Compos. Math. 145 (2009), 1035-1079. MR2521253(2010h:18021) 
7. W. Crawley-Boevey, On the exceptional fibres of Kleinian singularities, Amer. J. Math. 122 (2000), 1027-1037. MR1781930 (2001f:14009)

8. W. Crawley-Boevey, M. P. Holland, Noncommutative deformations of Kleinian singularities, Duke Math. J. 92 (1998), 605-635. MR1620538 (99f:14003)

9. C. Geiß, B. Leclerc, J. Schröer, Partial flag varieties and preprojective algebras, Ann. Inst. Fourier (Grenoble) 58 (2008), 825-876. MR2427512 (2009f:14104)

10. C. Geiß, B. Leclerc, J. Schröer, Kac-Moody groups and cluster algebras, Adv. Math. 228 (2011), 329-433.

11. J.-B. Hiriart-Urruty, C. Lemaréchal, Fundamentals of convex analysis, Grundlehren Text Editions, Springer-Verlag, 2001. MR.1865628 (2002i:90002)

12. J. Kamnitzer, The crystal structure on the set of Mirković-Vilonen polytopes, Adv. Math. 215 (2007), 66-93. MR2354986 (2009a:17021)

13. J. Kamnitzer, Mirković-Vilonen cycles and polytopes, Ann. of Math. 171 (2010), 245-294. MR2630039 (2011g:20070)

14. J. Kamnitzer, C. Sadanand, Modules with 1-dimensional socle and components of Lusztig quiver varieties in type A, preprint, arXiv:1009.0272.

15. M. Kashiwara, On crystal bases, Representations of groups (Banff, AB, 1994), pp. 155-197, CMS Conf. Proc., vol. 16, American Mathematical Society, 1995. MR1357199 (97a:17016)

16. M. Kashiwara, Y. Saito, Geometric construction of crystal bases, Duke Math. J. 89 (1997), 9-36. MR1458969 (99e:17025)

17. Y. Kimura, Affine quivers and crystal bases, Master thesis, University of Kyoto (Japan), 2007.

18. G. Lusztig, Canonical bases arising from quantized enveloping algebras, J. Amer. Math. Soc. 3 (1990), 447-498. MR.1035415 (90m:17023)

19. G. Lusztig, Canonical bases arising from quantized enveloping algebras II, Progr. Theoret. Phys. Suppl. 102 (1990), 175-201. MR1182165 (93g:17019)

20. G. Lusztig, Quivers, perverse sheaves, and quantized enveloping algebras, J. Amer. Math. Soc. 4 (1991), 365-421. MR.1088333 (91m:17018)

21. G. Lusztig, Braid group action and canonical bases, Adv. Math. 122 (1996), 237-261. MR.1409422 (98g:17019)

22. G. Lusztig, On quiver varieties, Adv. Math. 136 (1998), 141-182. MR1623674 (2000c:16016)

23. G. Lusztig, Semicanonical bases arising from enveloping algebras, Adv. Math. 151 (2000), 129-139. MR:1758244 (2001e:17033)

24. H. Nakajima, Instantons on ALE spaces, quiver varieties, and Kac-Moody algebras, Duke Math. J. 76 (1994), 365-416. MR1302318 (95i:53051)

25. H. Nakajima, Quiver varieties and Kac-Moody algebras, Duke Math. J. 91 (1998), 515-560. MR.1604167 (99b:17033)

26. C. Riedtmann, Algebren, Darstellungsköcher, Überlagerungen und zurück, Comment. Math. Helv. 55 (1980), 199-224. MR0576602 (82k:16039)

27. C. M. Ringel, PBW-bases of quantum groups, J. Reine Angew. Math. 470 (1996), 51-88. MR:1370206 (97d:17009)

28. C. M. Ringel, The preprojective algebra of a quiver, Algebras and modules, II (Geiranger, 1996), pp. 467-480, CMS Conf. Proc., vol. 24, American Mathematical Society, 1998. MR1648647 (99i:16031)

29. Y. Saito, $P B W$ basis of quantized universal enveloping algebras, Publ. Res. Inst. Math. Sci. 30 (1994), 209-232. MR 1265471 (95e:17021)

30. Y. Saito, Crystal bases and quiver varieties, Math. Ann. 324 (2002), 675-688. MR.1942245 (2004a:17023)

31. Y. Saito, personal communication, November 2010.

32. A. Savage, Geometric and combinatorial realizations of crystals of enveloping algebras, Lie algebras, vertex operator algebras and their applications (Raleigh, NC, 2005), pp. 221-232, Contemp. Math., vol. 442, American Mathematical Society, 2007. MR2372565 (2009d:17024) 
Institut de Recherche Mathématique Avancée, Université de Strasbourg et CNRS, 7 rue René Descartes, 67084 Strasbourg Cedex, France

E-mail address: p.baumann@unistra.fr

Department of Mathematics, University of Toronto, Bahen Centre, 40 St. George St., Toronto, Ontario, Canada ON M5S 2E4

E-mail address: jkamnitz@math.toronto.edu 